\title{
High-Affinity Alkynyl Bisubstrate Inhibitors of Nicotinamide N-Methyltransferase (NNMT)
}

\author{
SUPPORTING INFORMATION PART 3
}

Rocco L. Policarpo ${ }^{\mathrm{a}}$, Ludovic Decultot ${ }^{\mathrm{a}}$, Elizabeth May ${ }^{\mathrm{b}}$, Petr Kuzmičc ${ }^{\mathrm{c}}$, Sam Carlson ${ }^{\mathrm{b}}$, Danny Huang $^{\mathrm{a}}$, Vincent $\mathrm{Chu}^{\mathrm{a}, 1}$, Brandon Wright ${ }^{\mathrm{b}, 2}$, Saravanakumar Dhakshinamoorthy ${ }^{\mathrm{d}}$, Aimo Kannt $^{\mathrm{e}}$, Shilpa Rani ${ }^{\mathrm{d}}$, Sreekanth Dittakavi ${ }^{\mathrm{d}}$, Joseph Panarese ${ }^{\mathrm{a}, 3}$, Rachelle Gaudet ${ }^{\mathrm{b}}$, Matthew D. Shair*a

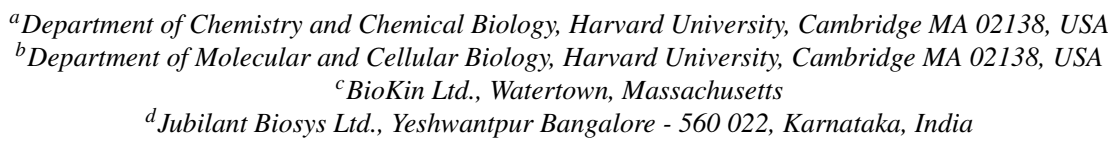

\begin{abstract}
This Supporting Information document contains a detailed description of all enzyme-kinetics analyses performed in order to characterize the 35 NNMT inhibitors listed in the main manuscript. In addition, we document detailed substrate-kinetic properties of NNMT under the conditions of the inhibitor screening assay. In particular, the fluorescence signal generated in the NNMT assay is strongly nonlinear due to two separate factors, namely, product inhibition and partial enzyme deactivation. Partial NNMT deactivation appears to proceed by a first-order decay of the Michaelis complex.
\end{abstract}

Key words:

nicotinamide N-methyltransferase; inhibition; bisubstrate analogs; molecular mechanism; kinetics; slow-binding; tight-binding

\section{Contents}

1 Substrate kinetic properties of SAM $\quad$ S3

1.1 Raw experimental data . . . . . . . . . . . . . . . . . . . . . . . . . . . . .

1.2 Theoretical models . . . . . . . . . . . . . . . . . S S4

1.2.1 Model MM . . . . . . . . . . . . . . . . . . S5

1.2.2 Model MM+pi . . . . . . . . . . . . . . . . . S5

\footnotetext{
${ }^{*}$ Corresponding author

${ }^{1}$ Current address: Department of Cell Biology, Harvard Medical School, 240 Longwood Ave., Boston, MA 02115, USA

${ }^{2}$ Current address: Department of Chemistry, University of California, Berkeley, CA 94720, USA

${ }^{3}$ Current address: Enanta Pharmaceuticals, 500 Arsenal St., Watertown, MA 02472, USA
} 
1.2.3 Model MM+ed . . . . . . . . . . . . . . . . . . . . . . . . . . . S5

1.2 .4 Model MM+pi+ed . . . . . . . . . . . . . . . . . . . . . . S6

1.2 .5 The regression equation $\ldots \ldots \ldots \ldots \ldots \ldots$. . . . . . . . . . 6

1.3 Results . . . . . . . . . . . . . . . . . . . . . S6

1.3.1 Representative example: Replicate R1 . . . . . . . . . . . . . S6

1.3.2 Model discrimination analysis $\ldots \ldots \ldots \ldots \ldots \ldots$. . . . . . . .

2 Substrate kinetic properties of quinoline $\quad \mathbf{S 1 0}$

2.1 Determination of initial rates . . . . . . . . . . . . . . . . S10

2.2 Determination of the apparent Michaelis constant $\ldots \ldots \ldots \ldots$. . . . S11

3 A general steady-state initial rate equation for bisubstrate inhibitors $\quad$ S12

3.1 Postulated kinetic mechanism . . . . . . . . . . . . . . . . . . . . . . . S12

3.2 The steady-state rate equation $\ldots \ldots \ldots \ldots \ldots \ldots$. . . . . . . . . . . . . . .

4 Inhibition kinetics: Theory and methods $\quad$ S16

4.1 Raw experimental data . . . . . . . . . . . . . . . . . S16

4.2 Theoretical models . . . . . . . . . . . . . . . . . . . . . . . . . S17

4.2.1 The regression equation . . . . . . . . . . . . . . . S17

4.2.2 Model R1S: "slow-binding" . . . . . . . . . . . . . . . . . . . . S17

4.2.3 Model R1F: "fast-binding" . . . . . . . . . . . . . . . . . . . . S18

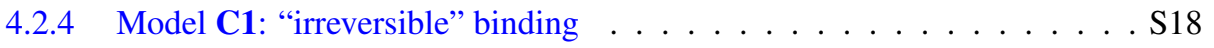

4.3 Two methods of handling substrate kinetic constants $\ldots \ldots \ldots \ldots \ldots$. . . S18

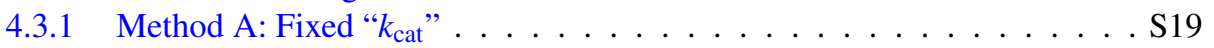

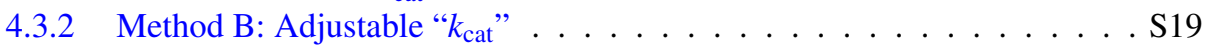

5 Inhibition kinetics: Results $\quad$ S19

5.1 Example 1: Compound 18, "fast-binding" . . . . . . . . . . . . . . . . . S19

5.1 .1 Model discrimination analysis . . . . . . . . . . . . . . . S20

5.1.2 Model-invariance of the inhibition constant . . . . . . . . . . . S21

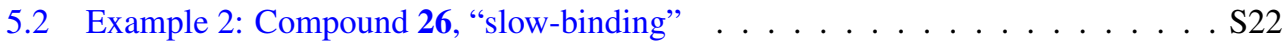

5.2 .1 Model discrimination analysis . . . . . . . . . . . . . . . S23

5.2.2 Confidence intervals for inhibition rate constants . . . . . . . . . . . S24

5.2.3 Distribution of enzyme species . . . . . . . . . . . . . . . . . . S24

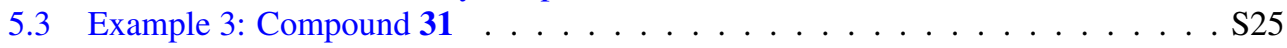

5.3.1 Model discrimination analysis . . . . . . . . . . . . . . S25

5.3.2 Reproducibility and model-invariance of inhibition constants . . . . . . S26

5.4 Potency ranking of NS1 analogs . . . . . . . . . . . . . . . . . S27

5.4 .1 Method A . . . . . . . . . . . . . . . . . . . . . S27

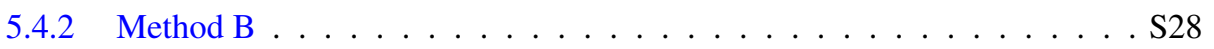

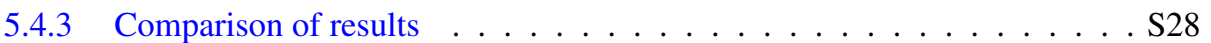

$\begin{array}{lr}\text { References } & \text { S28 }\end{array}$

$\begin{array}{ll}\text { Appendix } & \text { S31 }\end{array}$ 
A DynaFit scripts $\quad$ S31

A.1 Global fit of SAM substrate kinetic data . . . . . . . . . . . . . . S31

A.2 Local fit of quinoline substrate kinetic data . . . . . . . . . . . . . . . . S33

A.3 Determination of the apparent Michaelis constant for quinoline . . . . . . . . . . S34

A.4 Global fit of inhibition data, $\mathrm{Cpd}$ 31, Rep $1 \ldots \ldots$. . . . . . . . . S35

B Systems of ODEs automatically derived by DynaFit S38

B.1 Substrate kinetics of SAM . . . . . . . . . . . . . . . S38

B.1.1 Model MM . . . . . . . . . . . . . . . . . . . . S38

B.1.2 Model MM+pi . . . . . . . . . . . . . . . . . . . . . . . . . . . . . . . . . . . . . .

B.1.3 Model MM+ed . . . . . . . . . . . . . . . . . . . . . . . . S39

B.1.4 Model MM+pi+ed . . . . . . . . . . . . . . . . . . . S39

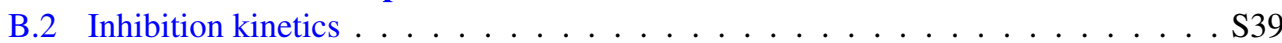

B.2.1 Models R1S and R1F . . . . . . . . . . . . . . . S39

B.2.2 Model C1 . . . . . . . . . . . . . . . . . . . S40

C Model-selection results $\quad$ S41

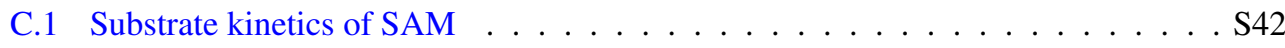

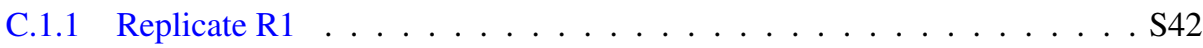

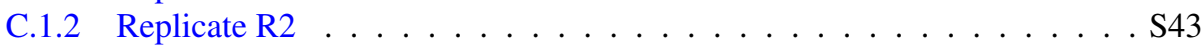

C.1.3 Replicate R3 . . . . . . . . . . . . . . . . . S44

C.2 Inhibitor kinetics of compound $18 \ldots \ldots \ldots \ldots \ldots$. . . . . . . . . . . . . . . . . . . . . . . .

C.3 Inhibitor kinetics of compound $26 \ldots \ldots \ldots \ldots \ldots \ldots$. . . . . . . . . . . . . . . . . . . . . . . . . . . . . .

C.4 Inhibitor kinetics of compound $31 \ldots \ldots \ldots \ldots$. . . . . . . . . . . . . . . . . . . . . . . .

C.4.1 Replicate 1 . . . . . . . . . . . . . . . . . . . S47

C.4.2 Replicate 2 . . . . . . . . . . . . . . . . . . . S48

C.4.3 Replicate $3 \ldots \ldots \ldots \ldots . \ldots . \ldots$. . . . . . . . . . . . . . . . . . . .

\section{Substrate kinetic properties of SAM}

In this section we describe the data-analytic procedure that was used to establish the basic kinetic properties of the substrate SAM, which were subsequently used as fixed model parameters in the analysis of the inhibition data.

\subsection{Raw experimental data}

The raw experimental data are shown in Figure S1. Three replicated experiments were performed and analyzed; two of the three replicates are shown. The reaction progress curves (fluorescence changes over time) were strongly nonlinear, which necessitated a choice of an appropriate nonlinear fitting model. 


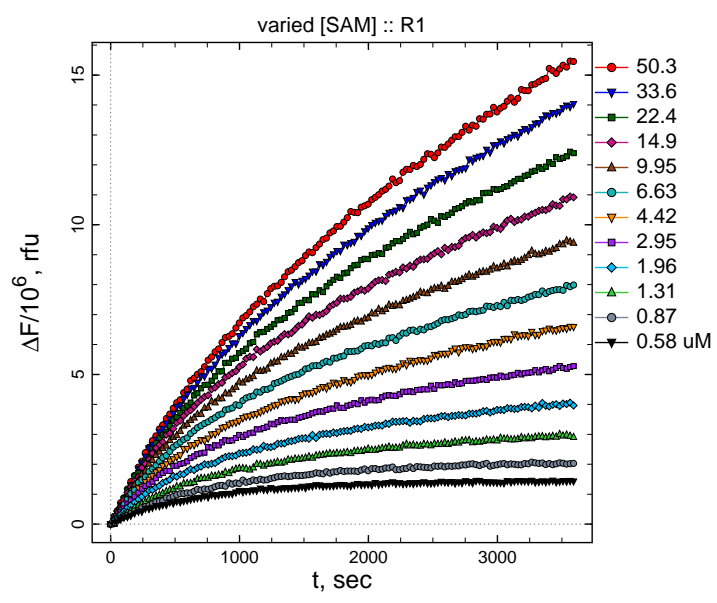

Replicate $1 / 3$

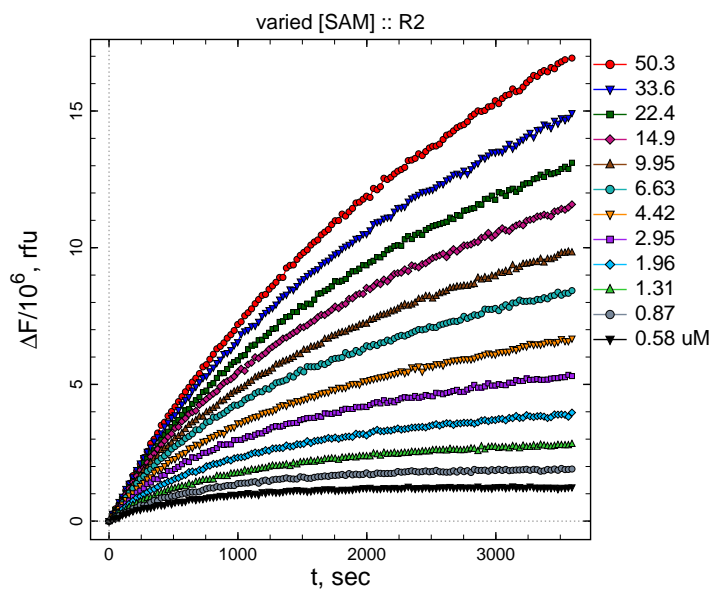

Replicate $2 / 3$

Figure S1: Raw experimental data from assays of NNMT conducted at $100 \mu \mathrm{M}$ quinoline and 33 nM enzyme.

\subsection{Theoretical models}

In order to fully explain the strong nonlinearity of the reaction progress curves, as illustrated in Figure S1, we have devised two sets of mechanistic models. In a preliminary round of kinetic analysis (results not shown) we hypothesized that the nonlinear reaction time course could be caused by any possible combination of the following factors:

1. substrate depletion;

2. product inhibition;

3. enzyme deactivation caused by the free enzyme E decaying via a first-order process;

4. enzyme deactivation caused by the Michaelis complex E.S decaying via a first-order process.

5. enzyme deactivation caused by both the free enzyme E and the Michaelis complex E.S decaying via a first-order processes.

The choice of multiple possible pathways for enzyme deactivation was informed by our previously published results [1]; see also [2] for a more detailed discussion. In this preliminary round of model discrimination analysis, we were able to unambiguously exclude the possibility that the free enzyme E undergoes deactivation. However, denaturation of the Michaelis complex E.S could not be excluded. In a follow-up refinement analysis we therefore set up a model discrimination analysis using four candidate models:

1. Model MM: The simple Michaelis-Menten kinetic mechanism, which accounts only for substrate depletion.

2. Model MM+pi: The Michaelis-Menten mechanism accompanied by product inhibition.

3. Model MM+ed: The Michaelis-Menten mechanism accompanied by enzyme deactivation via the E.S complex. 
4. Model MM+pi+ed: The Michaelis-Menten mechanism accompanied by product inhibition and by enzyme deactivation via the E.S complex.

The four candidate kinetic were each mathematically represented by an appropriate set of first-order ordinary differential equations automatically derived by the software package DynaFit $[3,4]$.

\subsubsection{Model MM}

$$
\begin{aligned}
& \mathrm{E}+\mathrm{S} \rightleftharpoons \mathrm{E} . \mathrm{S} \quad: \quad \overrightarrow{k_{\mathrm{a} . \mathrm{S}}} \leftarrow \\
& \mathrm{E} . \mathrm{S} \rightarrow \mathrm{E}+\mathrm{P} \quad: k_{\mathrm{d} . \mathrm{P}}
\end{aligned}
$$

In this kinetic mechanism, $k_{\mathrm{a} . \mathrm{S}}=10^{7} \mathrm{M}^{-1} \mathrm{~s}^{-1}$ is the second-order bimolecular association rate constant for the formation of the enzyme-substrate complex; it was held as a fixed parameter in the fit of the experimental data where SAM was the varied component; $k_{\mathrm{d} . \mathrm{S}}$ (unit: $\mathrm{s}^{-1}$ ) is the dissociation rate constant for the decomposition of the Michaelis complex into constituent components; and $k_{\mathrm{d} . \mathrm{P}}$ (unit: $\mathrm{s}^{-1}$ ) first-order rate constant for the formation of the monitored reaction product $P$. In the classical notation this particular rate constant would be identified as the catalytic constant " $k_{\text {cat }}$ ".

The corresponding system of differential equations automatically derived by DynaFit [4] is listed in Appendix B.1.1

\subsubsection{Model $\mathbf{M M + p i}$}

$$
\begin{aligned}
& \mathrm{E}+\mathrm{S} \rightleftharpoons \mathrm{E} . \mathrm{S} \quad: \quad \overrightarrow{k_{\mathrm{a} . \mathrm{S}}} \quad \overleftarrow{k_{\mathrm{d} . \mathrm{S}}} \\
& \mathrm{E} . \mathrm{S} \rightarrow \mathrm{E}+\mathrm{P}: k_{\mathrm{d} . \mathrm{P}} \\
& \mathrm{E}+\mathrm{P} \rightleftharpoons \mathrm{E} . \mathrm{P} \quad: k_{\mathrm{a} . \mathrm{Pi}} k_{\mathrm{d} . \mathrm{Pi}}
\end{aligned}
$$

In this kinetic mechanism, $k_{\mathrm{a} . \mathrm{Pi}}=10^{7} \mathrm{M}^{-1} \mathrm{~s}^{-1}$ is the second-order bimolecular association rate constant for the formation of the enzyme-product complex accounting for possible product inhibition; it was held as a fixed parameter; $k_{\mathrm{d} . \mathrm{Pi}}\left(\right.$ unit: $\left.\mathrm{s}^{-1}\right)$ is the dissociation rate constant for the decomposition of the inhibited E.P complex into constituent components.

The corresponding system of differential equations automatically derived by DynaFit [4] is listed in Appendix B.1.2

\subsubsection{Model MM+ed}

$$
\begin{array}{rllll}
\mathrm{E}+\mathrm{S} & \rightleftharpoons \mathrm{E} . \mathrm{S} & : & k_{\mathrm{a} . \mathrm{S}} & k_{\mathrm{d} . \mathrm{S}} \\
\mathrm{E} . \mathrm{S} \rightarrow \mathrm{E}+\mathrm{P} & : & k_{\mathrm{d} . \mathrm{P}} & \\
\mathrm{E} . \mathrm{S} \rightarrow \mathrm{F} & : & k_{\mathrm{ES}}
\end{array}
$$

In this kinetic mechanism, $k_{\mathrm{ES}}$ is the first-order rate constant (unit: $\mathrm{s}^{-1}$ ) for the irreversible denaturation of the Michaelis complex [1].

The corresponding system of differential equations automatically derived by DynaFit [4] is listed in Appendix B.1.3 


\subsubsection{Model $\mathbf{M M + p i + e d ~}$}

$$
\begin{array}{rllll}
\mathrm{E}+\mathrm{S} & \rightleftharpoons \mathrm{E} . \mathrm{S} & : & k_{\mathrm{a} . \mathrm{S}} & k_{\mathrm{d} . \mathrm{S}} \\
\mathrm{E} . \mathrm{S} & \rightarrow \mathrm{E}+\mathrm{P} & : & k_{\mathrm{d} . \mathrm{P}} \\
\mathrm{E}+\mathrm{P} & \rightleftharpoons \mathrm{E} . \mathrm{P} & : & k_{\mathrm{a} . \mathrm{Pi}} & k_{\mathrm{d} . \mathrm{Pi}} \\
\mathrm{E} . \mathrm{S} & \rightarrow \mathrm{F} & : & k_{\mathrm{ES}} &
\end{array}
$$

This final kinetic mechanism combines the essential features of product inhibition and enzyme deactivation occurring simultaneously.

The corresponding system of differential equations automatically derived by DynaFit [4] is listed in Appendix B.1.4

\subsubsection{The regression equation}

The fitting function (model equation) for each individual data set is defined by Eqn (S1),

$$
F(t)=F_{0}+\sum_{i=1}^{n_{S}} r_{i} c_{i}(t)
$$

where

$F(t) \quad$ the experimental signal observed at time $t$

$F_{0} \quad$ offset on the signal axis (a property of the instrument)

$n_{S} \quad$ number of unique molecular species participating in the reaction mechanism

$c_{i}(t)$ the concentration of the $i$ th species at time $t$

$r_{i} \quad$ the molar response coefficient of the $i$ th species

The concentrations of these molecular species at time $t$ are computed from their initial concentrations (at time zero, $t=0$ ) by solving an initial-value problem defined by a system of simultaneous first-order Ordinary Differential Equations (ODEs) listed above.

In the global fit [5] of combined progress reaction progress curves, the rate constants were treated as globally adjustable model parameters, as was the molar response coefficient of the fluorogenic product, P. In contrast, each kinetic trace in the global data set was associated with its own locally adjustable offset on the signal axis.

\subsection{Results}

\subsubsection{Representative example: Replicate R1}

Figure $S 2$ represents in panel (a) the overlay of experimental data (replicate 1/3) spanning the first 20 minutes of the assay and the theoretical model curves generated from model $\mathbf{M M + p i + e d ~}$ using the best-fit values of rate constants listed in the table immediately below. The best-fit values of the locally adjusted baseline offsets $\left(F_{0}\right.$ in Eqn $\left.(\mathrm{S} 1)\right)$ are omitted for brevity.

\begin{tabular}{llrrr}
\hline Model & Parameter & Value & Low & High \\
\hline MM+Pi+Ed & $k_{\text {d.S }}, \mathrm{s}^{-1}$ & 8 & 6.07 & 10 \\
& $k_{\text {d.P }}, \mathrm{s}^{-1}$ & 0.0301 & 0.0283 & 0.0319 \\
& $k_{\text {d.Pi }}, \mathrm{s}^{-1}$ & 0.984 & 0.701 & 1.32 \\
& $k_{\mathrm{ES}}, \mathrm{s}^{-1}$ & 0.000678 & 0.00063 & 0.000727 \\
\hline
\end{tabular}




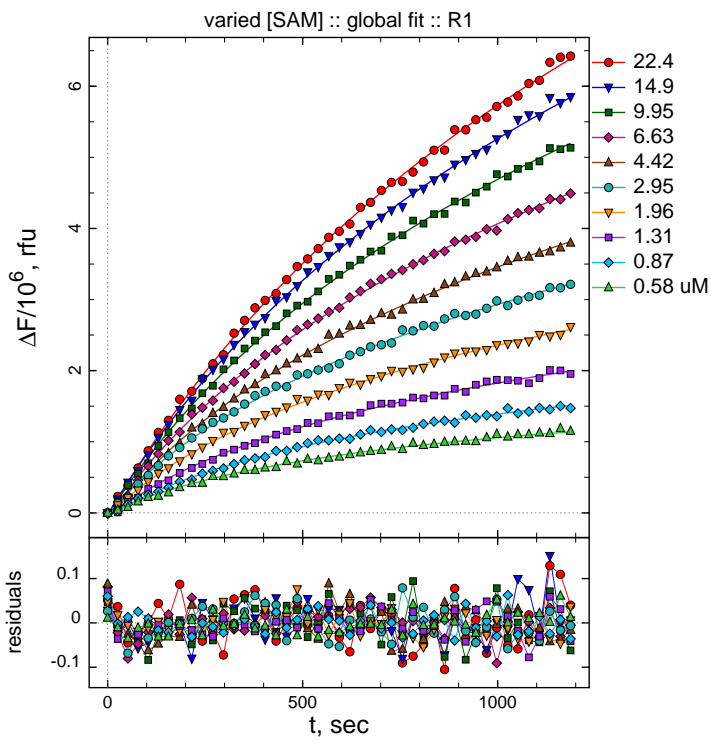

(a)

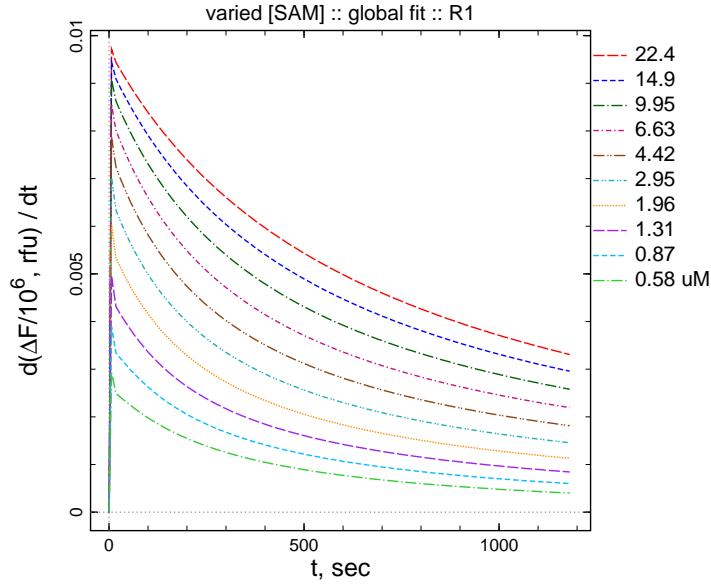

(b)

Figure S2: Global nonlinear least-squares fit of combined control progress curves (zero inhibitor, varied SAM) to model MM+pi+ed, i.e., Michaelis-Menten mechanism accompanied by product inhibition and enzyme deactivation.

Panel (b) in Figure $S 2$ displays the changes in instantaneous reaction rates over the course of the first 20 minutes of the assay. The inventors of this particular enzyme assay [6] recommended a linear fit of the first 3 minutes, in order to determine initial reaction rates. This recommendation assumes that the reaction rate remains essentially unchanged over the given time period.

However, as can be seen in panel (b) of Figure S2, under the particular experimental conditions employed in this laboratory the instantaneous reaction rates change by as much as $25 \%-$ $33 \%$ between $t=0$ and $t=3 \mathrm{~min}$. This means that a linear fit of the 3 -minute reaction progress is unsuitable and that a nonlinear regression procedure is necessary to quantify the enzymatic activity. 


\begin{abstract}
Note
The authors of the fluorescence assay [6] do not specify which variant of hNNMT they used. A relevant report [7] cited in the original publication presents both a wild-type (wt) variant and a triple-mutant (tm) variant used for crystallography. These enzymes have differential activity (relative activity of wt-hNNMT is $86 \%$ that of tm-hNNMT). The tm-hNNMT variant contains three mutations (K100A/E101A/E103A) designed to aid crystallization by reducing the entropy of surface-exposed residues in a loop distant from the active site [7]. These surface mutations could render a more stable protein, changing the rate of first-order decay of the Michaelis complex E.S. In our assays we used the wild-type variant (wt-hNNMT), but it is possible that wt-hNNMT could be more sensitive and unstable than tm-hNNMT.
\end{abstract}

\title{
1.3.2. Model discrimination analysis
}

\section{Algorithm}

The optimal mechanistic model for SAM substrate kinetic was selected by the software package DynaFit [4], using the following generally applicable model-selection criteria:

1. For a given candidate model to be accepted as plausible, the confidence intervals for all model parameters determined by the profile- $t$ method of Bates and Watts [8-10] must be closed from both below and above at the given confidence level.

2. For a given plausible model to be identified as strongly preferred over other plausible candidate models, which also satisfy the acceptance criterion defined immediately above, one of two of the following criteria must hold:

(a) Non-continuous assays: The differences in the Akaike Information Criterion (AIC) $[11,12]$ and the Bayesian Information Criterion (BIC) $[12,13]$ must be greater than 5 .

(b) Continuous assays: The differences in relative sum of squared deviations must be greater than $5 \%$.

Note that the particular kinetic experiments conducted in this study belong into the category of continuous assays, where individual data points (time vs. fluorescence signal values) are not statistically independent and therefore the AIC and BIC model selection criteria cannot be used [14-16] in the usual manner.

\section{Results}

The auto-generated model discrimination analysis for replicates R1-R3 is shown in Appendix C.1.1-C.1.3. For all three independently replicated experiments, the preferred mechanistic model was MM+pi+ed, i.e., the Michaelis-Menten kinetic mechanism accompanied by both product inhibition and enzyme deactivation caused by a significant decay of the Michaelis complex ES. The results of model discrimination and parameter estimation are summarized in Table S1.

Note in Table S1 that the best-fit values of microscopic rate constants are well reproduced among the three replicated measurements. In particular, the geometric standard deviation is 


\begin{tabular}{lrrrrr}
\hline parameter & $\mathrm{R} 1$ & $\mathrm{R} 2$ & $\mathrm{R} 3$ & $\mathrm{GM}$ & $\mathrm{GSD}$ \\
\hline$k_{\mathrm{d} . \mathrm{S}}, \mathrm{s}^{-1}$ & 8.0 & 12.8 & 15.8 & 11.8 & 1.4 \\
$k_{\mathrm{kd} . \mathrm{P}}, \mathrm{s}^{-1}$ & 0.030 & 0.038 & 0.041 & 0.036 & 1.2 \\
$k_{\mathrm{kd} . \mathrm{Pi}}, \mathrm{s}^{-1}$ & 0.98 & 2.69 & 3.29 & 2.06 & 1.9 \\
$k_{\mathrm{k} . \mathrm{ES}}, \mathrm{s}^{-1}$ & 0.00068 & 0.00054 & 0.00054 & 0.00058 & 1.1 \\
\hline
\end{tabular}

Table S1: Substrate kinetic properties of SAM. GM = geometric mean from replicates R1-R3 $(n=3) ; \mathrm{GSD}=$ geometric standard deviation.

lower than 2.0 for all four rate constants of interest, which means that the characteristic range of the relevant model parameter is within the factor of 2 in either direction (lower and higher relative to the geometric mean).

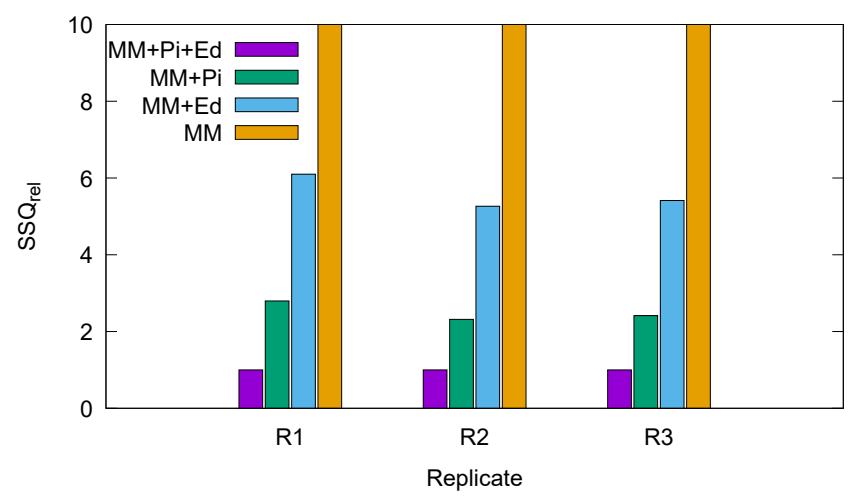

Figure S3: Relative residual sum of squared deviations obtained in the analysis of SAM substrate kinetic data in the fit to the four candidate fitting models. All three $S S Q_{\mathrm{r}}$ values for the model MM are all greater than 20 and are off-scale in this illustration.

The statistical preference for model MM+pi+ed is illustrated graphically in Figure S3. The relative sum-of-squares $\left(S S Q_{\mathrm{r}}\right)$ values were extracted from the detailed model discrimination report in Appendix C.1.1-C.1.3. The $S S Q_{\mathrm{r}}$ value for the runner-up model MM+pi (MichaelisMenten kinetics accompanied by product inhibition) was at least twice as high as the $S S Q_{\mathrm{r}}$ for the optimal model MM+pi+ed (set to 1.0 by default).

The model MM+ed (Michaelis-Menten kinetics accompanied by enzyme deactivation) is associated with the same number of adjustable rate constants as the runner-up model MM+pi but, at the same time, model MM+ed produced a much higher value of $S S Q_{\mathrm{r}}>5$. Thus enzyme deactivation alone cannot account for the deviation of SAM progress curves from the simple Michaelis-Menten kinetic mechanism.

The base model MM (pure Michaelis-Menten kinetics) was associated with extremely high values of $S S Q_{\mathrm{r}}>20$ which, for clarity, are off-scale in Figure $S 3$ and it can therefore be unambiguously excluded from consideration. The deviation of the "best-fit" model curves generated from model MM were also clearly detectable by simple visual inspection of the experimental data overlaid on the model curves (results not shown). 
It should be noted that strictly speaking the $S S Q_{\mathrm{r}}$ values displayed in Figure $S 3$ have only informative value, because the number of adjustable model parameters (rate constants) differs between candidate models. However, previously published empirical evidence [14] strongly suggests that $S S Q_{\mathrm{r}}>2.0$ is highly indicative of model discrimination power.

\section{Substrate kinetic properties of quinoline}

In this section we describe the determination of the apparent Michaelis constant of quinoline, $K_{\mathrm{mQ}}^{(\mathrm{app})}$, at the screening concentration of $[\mathrm{SAM}]=10 \mu \mathrm{M}$. This value is subsequently utilized to adjust the apparent inhibition constants to account for the competitive mechanism of inhibition of bisubstrate analogs.

\subsection{Determination of initial rates}

NNMT (100 nM) was assayed at $10 \mu \mathrm{M}$ SAM in the absence of inhibitors. Quinoline concentration was varied from 10 to $120 \mu \mathrm{M}$. The individual reaction progress curves were fit to the integrated rate equation based on the Lambert Omega function [17] in order to determine initial reaction rates. See ref. [17] for further mathematical details. The requisite DynaFit [4] that was used to perform this kinetic analysis is listed in Appendix A.2. The results of fit are summarized graphically in Figure 54 , for replicate 1/3.

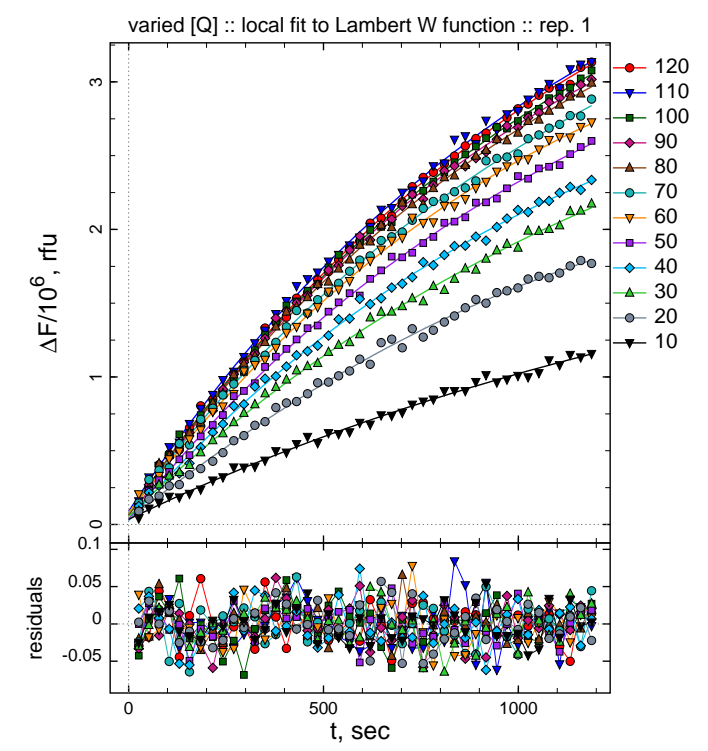

(a) Data and model overlay

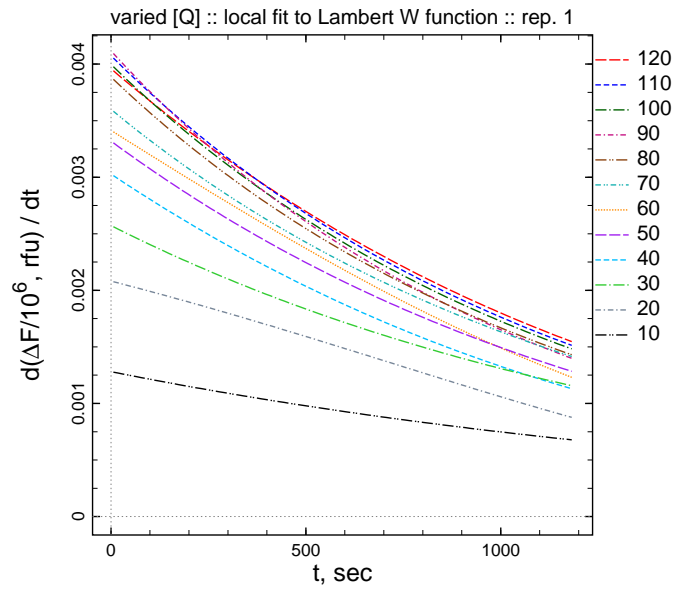

(b) Instantaneous reaction rates

Figure S4: Least-squares fit of individual progress curves ("local" fit) to the theoretical model based on the Lambert Omega function [17] to determine initial reaction rates in dependence on quinoline concentration.

Note in Figure $S 4$ that the reaction time course is markedly nonlinear, as is evident from panel (b) displaying the instantaneous reaction rates. This nonlinearity necessitated the use of a 
nonlinear regression model, as opposed to the standard linear fit of the "initial portion" in each individual reaction progress curve.

The goodness-of-fit to the Lambert Omega function [17] is satisfactory, as is evidenced by the random distribution of residuals of fit, bottom pane labeled "residuals" in panel (a) of Figure $S 4$. The residual distribution is not showing any particular non-random pattern.

\subsection{Determination of the apparent Michaelis constant}

The initial reaction rates determined as described in section 2.1 were pooled, averaged, and fit to the Michaelis-Menten kinetic model represented symbolically as shown in the DynaFit [4] script listed in Appendix A.3. The results of fit are summarized graphically in Figure S5.

The best-fit parameter values are listed in the table immediately below. The "high" and "low" columns contain the upper and lower limits, respectively, of the $95 \%$ confidence interval determined by the profile- $t$ method of Bates and Watts $[8,9]$.

\begin{tabular}{llrcrrrr}
\hline$\#$ & parameter & initial & final \pm std.err. & cv, \% & low & high & note \\
\hline 1 & $K_{\mathrm{m}}, \mu \mathrm{M}$ & 10 & $32.3 \pm 1.5$ & 4.6 & 29.1 & 35.8 & \\
2 & $k_{\text {cat }}, \mathrm{s}^{-1}$ & 0.1 & $53.54 \pm 0.84$ & 1.6 & 51.76 & 55.47 & \\
\hline
\end{tabular}

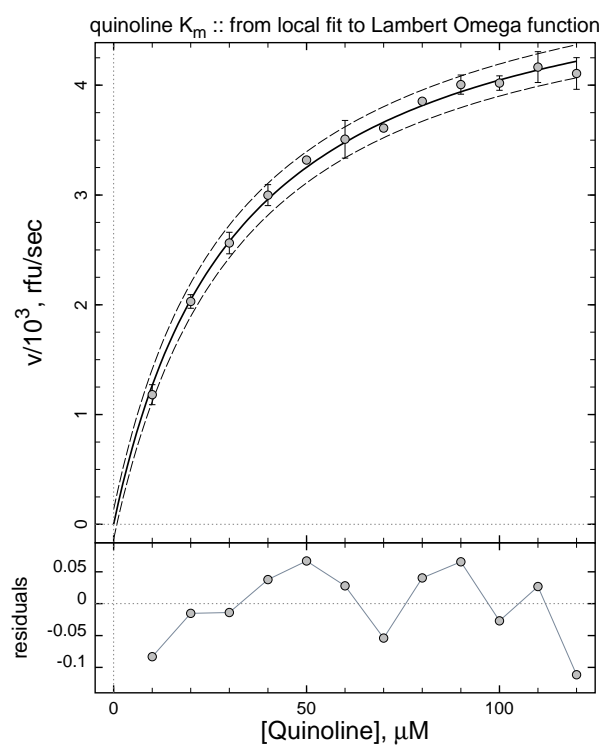

Figure S5: Least-squares fit of initial reaction rates from quinoline assay to the MichaelisMenten reaction mechanism represented symbolically as shown in the DynaFit script listed in Appendix A.3. For details see text.

The results show that the apparent Michaelis constants of quinoline at the inhibitor screening concentration of $[\mathrm{SAM}]=10 \mu \mathrm{M}$ is equal to $K_{\mathrm{mQ}}^{(\mathrm{app})}=32 \mu \mathrm{M}$. This result was utilized below in 
our assessment of "apparent" vs. "true" inhibition constants determined as is explained in section 4.

\section{A general steady-state initial rate equation for bisubstrate inhibitors}

In this section we describe the use of the software package DynaFit [4] for the automatic derivation of the initial rate equation for any bisubstrate inhibitor acting on an enzyme that follows the "Ordered Bi Bi" kinetic mechanism [18], also known as "compulsory-order ternarycomplex" mechanism [19]. The "Ordered Bi Bi" mechanism is relevant because Loring \& Thompson [20] had recently demonstrated that a triple mutant of the recombinant human NNMT enzyme follows it.

The purpose of this derivation is to elucidate any possible difference between "apparent" inhibition constants [21] and "true" inhibition constants [22], in particular under tight-binding experimental conditions employed in this study.

\subsection{Postulated kinetic mechanism}

We assume that inhibition of NNMT follows the steady-state kinetic mechanism shown in the reaction scheme immediately below.

$$
\begin{aligned}
& \mathbf{A}+\mathbf{B} \rightarrow \mathbf{P}+\mathbf{Q} \\
& \mathrm{E}+\mathrm{A} \rightleftharpoons \mathrm{E} . \mathrm{A} \quad: \overrightarrow{k_{1}} \leftarrow \\
& \mathrm{E} . \mathrm{A}+\mathrm{B} \rightleftharpoons \mathrm{E} . \mathrm{X} . \mathrm{Y}: k_{2} k_{-2} \\
& \text { E.X.Y } \rightarrow \text { E.Q }+ \text { P : } k_{3} \\
& \mathrm{E} . \mathrm{Q} \rightarrow \mathrm{E}+\mathrm{Q}: k_{4} \\
& \mathrm{E}+\mathrm{I} \rightleftharpoons \mathrm{E} . \mathrm{I} \quad: k_{5} \quad k_{-5}
\end{aligned}
$$

In this mechanistic scheme, $\mathbf{A}$ and $\mathbf{B}$ represent SAM and quinoline, respectively. In the first step, SAM (A) attaches to the free enzyme, E. In contrast, quinoline (B) does not interact with the free enzyme but binds instead only to the binary complex E.A. This is according to the results of a mechanistic study previously reported in ref. [20].

The symbol "E.X.Y" stands for the ternary complex in both its isomeric incarnations (i.e., $E A B \rightleftharpoons E P Q$ in the frequently used notation). The ternary complex releases methyl-quinoline (P) as the first product and finally $\mathrm{SAH}(\mathbf{Q})$ is released to complete the catalytic cycle and regenerate the free catalyst, E.

We assume that in the fifth step characterized by rate constants $k_{5}$ and $k_{-5}$ the inhibitor I binds to the free enzyme $\mathrm{E}$ (i.e., the apoenzyme), and that this is the only interaction between the inhibitor and the enzyme. This assumption is based on the fact that by definition bisubstrate analog inhibitors physically occlude the binding sites for both substrates and therefore when the inhibitor is bound neither of the substrates and/or products can bind at the same time.

In the input file for the software package DynaFit [4], the kinetic mechanism shown above is represented by using the following notation:

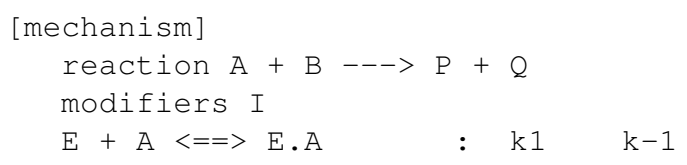




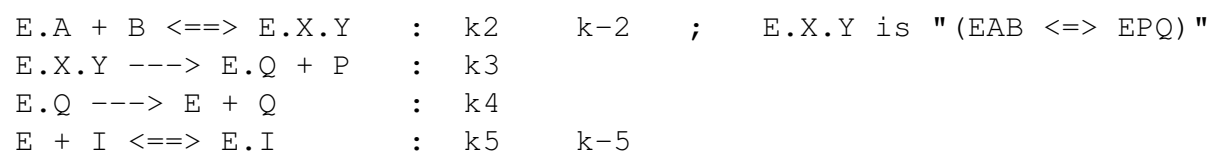

\subsection{The steady-state rate equation}

We used the software package DynaFit [4] to derive the relevant algebraic form of the "Morrison equation" [23] corresponding to the inhibition mechanism listed above in section 3.1. The requisite DynaFit script is listed below:

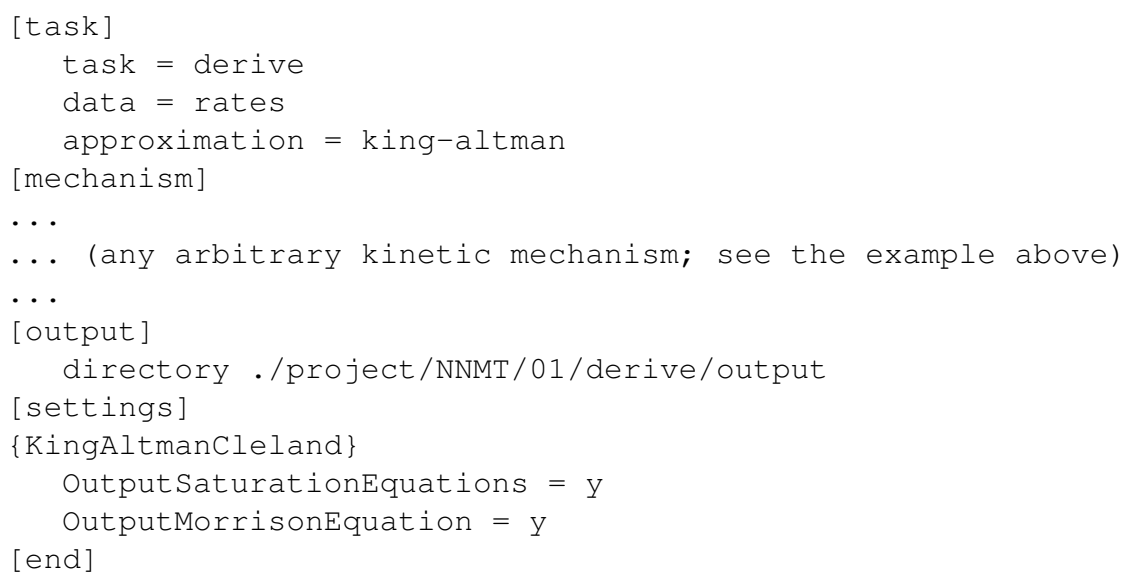

Note that the "Morrison equation" for tight-binding enzyme inhibition [21] is relevant because the enzyme concentration in our inhibitor screening assay was typically $100 \mathrm{nM}$, while at the same time many of the inhibition constants were in the same range or even significantly lower (sub-nanomolar in several cases).

\section{Note}

Morrison, on advice from W. W. Cleland (see the Acknowledgement section in ref. [23]), did not actually derive a steady-state initial rate equation that applies to any particular kinetic mechanism. Rather, he derived a general "template" formula that applies to nearly all kinetic mechanisms of inhibition (except partial inhibition mechanisms and inhibition mechanisms involving higher that 1:1 stoichiometry). Nor did Morrison provide any example how his generic "template" formula could be specialized for any given inhibition mechanism. The software package DynaFit [4] does include an algorithm for precisely this type of specialization, such that an actual rate equation is formed from the generic "template" derived by Morrison [23].

When the DynaFit package was presented with the script file listed above, the software automatically derived the "Morrison equation" for the postulated inhibition mechanism, as is shown in the system of equations immediately below: 


$$
\begin{aligned}
v & =v_{0} \frac{[\mathrm{E}]_{0}-[\mathrm{I}]_{0}-K_{\mathrm{i}}^{(\mathrm{app})}+\sqrt{\left([\mathrm{E}]_{0}-[\mathrm{I}]_{0}-K_{\mathrm{i}}^{(\mathrm{app})}\right)^{2}+4[\mathrm{E}]_{0} K_{\mathrm{i}}^{(\mathrm{app})}}}{2[\mathrm{E}]_{0}} \\
K_{\mathrm{i}}^{(\mathrm{app})} & =\frac{d_{0}}{d_{\mathrm{I}}} \\
d_{\mathrm{I}} & =\frac{1}{K_{\mathrm{dI}}}+\frac{K_{\mathrm{mA}}[\mathrm{B}]_{0}}{K_{\mathrm{dA}} K_{\mathrm{mB}} K_{\mathrm{dI}}} \\
v_{0}= & {[\mathrm{E}]_{0} \frac{N}{d_{0}} } \\
N= & k_{\mathrm{cat}} \frac{[\mathrm{A}]_{0}[\mathrm{~B}]_{0}}{K_{\mathrm{dA}} K_{\mathrm{mB}}} \\
d_{0}= & 1+\frac{[\mathrm{A}]_{0}}{K_{\mathrm{dA}}}+\frac{K_{\mathrm{mA}}[\mathrm{B}]_{0}}{K_{\mathrm{dA}} K_{\mathrm{mB}}}+\frac{[\mathrm{A}]_{0}[\mathrm{~B}]_{0}}{K_{\mathrm{dA}} K_{\mathrm{mB}}} \\
k_{\mathrm{cat}} & =\frac{k_{3} k_{4}}{k_{4}+k_{3}} \\
K_{\mathrm{mA}} & =\frac{k_{3} k_{4}}{k_{1}\left(k_{4}+k_{3}\right)} \\
K_{\mathrm{mB}} & =\frac{k_{4}\left(k_{-2}+k_{3}\right)}{k_{2}\left(k_{4}+k_{3}\right)} \\
K_{\mathrm{dA}} & =\frac{k_{-1}}{k_{1}} \\
K_{\mathrm{dI}} & =\frac{k_{-5}}{k_{5}}
\end{aligned}
$$

The above system of equations, auto-generated by the DynaFit software package, can be rearranged to express the apparent inhibition constant in a more compact form as is shown in Eqn (S13).

$$
K_{\mathrm{i}}^{(\mathrm{app})}=K_{\mathrm{dI}} \frac{K_{\mathrm{dA}} K_{\mathrm{mB}}+K_{\mathrm{mB}}[\mathrm{A}]_{0}+K_{\mathrm{mA}}[\mathrm{B}]_{0}+[\mathrm{A}]_{0}[\mathrm{~B}]_{0}}{K_{\mathrm{dA}} K_{\mathrm{mB}}+K_{\mathrm{mA}}[\mathrm{B}]_{0}}
$$

Eqn (S13) can be profitably analyzed in terms of the expected inhibition patterns (competitive, uncompetitive, noncompetitive, mixed-type) with respect to each of the two substrates. The limits of $K_{\mathrm{i}}^{(\mathrm{app})}$ at either $[\mathrm{A}]_{0}$ or $[\mathrm{B}]_{0}$ approaching zero are shown in Eqns (S14)-(S15). 


$$
\begin{array}{ll}
\lim _{[\mathrm{A}]_{0} \rightarrow 0} K_{\mathrm{i}}^{(\mathrm{app})}=K_{\mathrm{dI}} & \text { noncompetitive w.r.t. B } \\
\lim _{[\mathrm{B}]_{0} \rightarrow 0} K_{\mathrm{i}}^{(\mathrm{app})}=K_{\mathrm{dI}}\left(1+\frac{[\mathrm{A}]_{0}}{K_{\mathrm{dA}}}\right) & \text { competitive w.r.t. A }
\end{array}
$$

The assignment of competitive vs. noncompetitive patterns can be made in analogy to singlesubstrate systems analyzed by Cha [22]. According to Cha's analysis, the apparent inhibition constant for a competitive inhibitor will be higher than the "true" inhibition constant by the factor of $\left(1+[\mathrm{S}]_{0} / K_{\mathrm{M}}\right)$ (cf. [22, Eqn. (7)]). In contrast, the apparent inhibition constant for a noncompetitive inhibitor in a single-substrate system will not depend on the substrate concentration at all (cf. [22, Eqn. (9)]).

However, please note one important difference between Cha's Eqn (7) published in [22], including the multiplication factor $\left(1+[\mathrm{S}]_{0} / K_{\mathrm{M}}\right)$, and our Eqn (S15), including the multiplication factor $\left(1+[\mathrm{A}]_{0} / K_{\mathrm{dA}}\right)$. In Cha's case, the multiplication factor involves the Michaelis constant of the (in his case, single) substrate, whereas in our case the multiplication factor involves the dissociation equilibrium constant of the first attached substrate. Recall that Michaelis constants are always complex quantities encompassing many microscopic rate constants appearing in the given kinetic mechanism, whereas the dissociation equilibrium constant is always a simple ratio of the off rate constant divided by the on rate constant.

Taylor [24] derived a steady-state initial rate equation for a classical (as opposed to tightbinding) inhibitor interacting exclusively with the free enzyme in a bisubstrate system following the "Ordered Bi Bi" mechanism. The published algebraic derivation confirmed that such an inhibitor will show a competitive kinetic pattern with respect to the first attached substrate, and a noncompetitive inhibition pattern with respect to the second attached substrate (cf. [24, Eqns. 6.9 and 6.10, p. 74]).

The main purpose of the theoretical analysis presented in this section was as follows.

- All bisubstrate analog inhibitors (under either tight-binding or classical experimental conditions) acting on an enzyme following the "Ordered $\mathrm{Bi} \mathrm{Bi}$ " mechanism are noncompetitive with respect to the second attached substrate, in this case quinoline.

- Therefore, quinoline concentration in the NNMT assay has no effect on the apparent inhibition constant, $K_{\mathrm{i}}^{(\mathrm{app})}$.

- The apparent inhibition constant of all bisubstrate analog inhibitors acting on an enzyme following the "Ordered $\mathrm{Bi} \mathrm{Bi}$ " mechanism depend only on the concentration of the first attached substrate, such that $K_{\mathrm{i}}^{(\mathrm{app})}=K_{\mathrm{d}}\left(1+[\mathrm{A}]_{0} k_{\mathrm{on}} / k_{\text {off }}\right)$, where $k_{\text {on }}$ and $k_{\text {off }}$ are microscopic rate constants for the first attached substrate interacting with the apoenzyme.

- Therefore, if we can construct a suitable differential-equation model for the reaction progress explicitly incorporating $k_{\text {on }}$ and $k_{\text {off }}$ for the first attached substrate, the resulting best-fit values of the on/off rate constants for the (competitive) inhibitor will be the "true" rate constants and therefore their ratio will also be the "true" inhibition constant, i.e., the dissociation constant of the enzyme-inhibitor complex.

The above theoretical analysis proves that the best-fit values of on/off rate constants for bisubstrate analog inhibitors reported in this paper are "true" and not "apparent" rate constants. Therefore the off/on rate constant ratio is also the "true" inhibition constant, as opposed to an apparent inhibition constant. 


\section{Inhibition kinetics: Theory and methods}

\subsection{Raw experimental data}

Upon simple visual examination, the raw experimental data from our NNMT inhibition assays could be qualitatively classified into at least two distinct categories:

1. Strong nonlinearity due to "slow-binding" inhibition detectable by visual inspection.

2. Moderate nonlinearity, possibly with a "slow-binding" component and/or due to other factors.

In the first category were numerous kinetic experiments that resulted in prominently nonlinear reaction progress curves. The observed deviations from a linear time course were visibly more prominent than the gradual nonlinearity observed in positive control experiments, conducted in the absence of inhibitors (see for example Figure S1). This pronounced additional nonlinearity strongly suggests time-dependent i.e. "slow-binding” inhibition [25, 26].

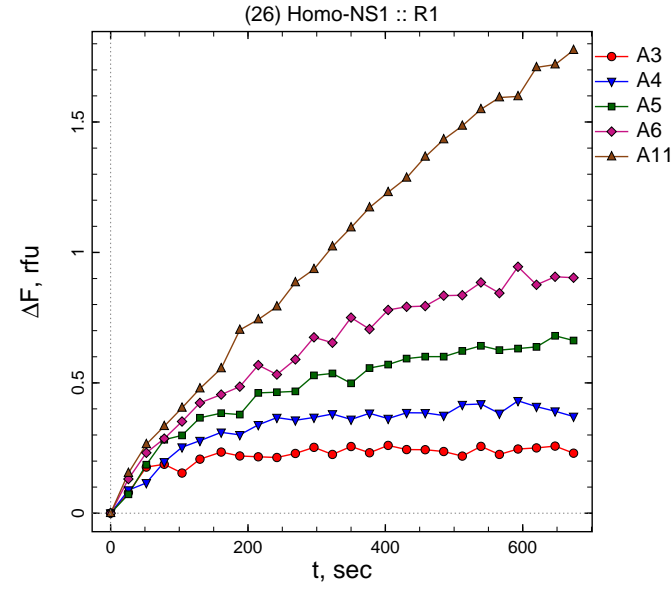

(a)

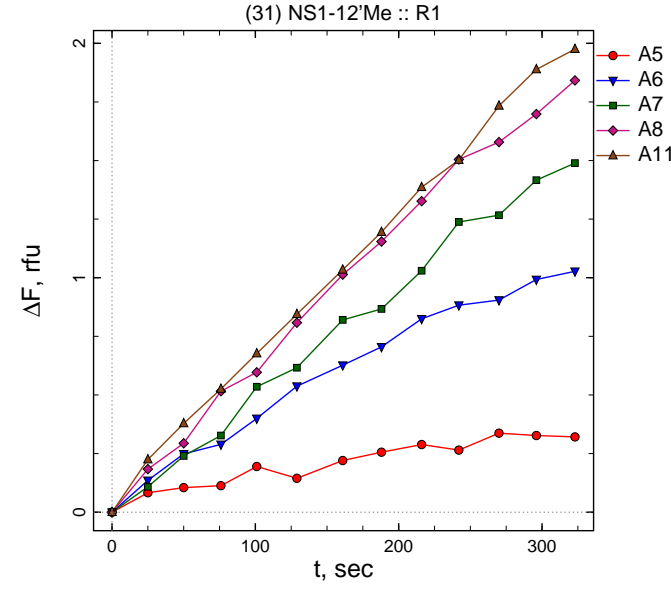

(b)

Figure S6: Raw experimental data from inhibition assays of NNMT. (a) - Compound 26, replicate 1, 33 nM NNMT; (b) - Compound 31, replicate 1, 100 nM NNMT. For details see text.

A typical example of clearly detectable "slow-binding" inhibition is illustrated in the lefthand panel (a) of Figure S6 (compound 26, replicate R1). The data set labels correspond to individual wells on a 96-well plate. The trace in well A11 was obtained in the absence of inhibitor; wells A3 - A6 correspond to inhibitor concentrations 293, 196, 131, and $87 \mathrm{nM}$, respectively.

The characteristic "slow-binding" inhibition pattern is most prominently illustrated by the kinetic trace labeled A4 (blue triangles down). The slope of a tangent to the progress curve drawn at time zero (i.e., the initial reaction rate at $[\mathrm{I}]=196 \mathrm{nM}$ ) would very closely approximate the uninhibited rate (i.e., the initial reaction rate at $[\mathrm{I}]=0$ ). The instantaneous rate (slope) subsequently changes over time, until, at approximately $t=5 \mathrm{~min}$, the reaction progress curve becomes nearly horizontal, corresponding to nearly complete inhibition. Thus, in this particular case, the inhibitory effect gradually developed over the course of approximately 5 minutes (hence "slow-binding"). 
A typical example of the other kind of progress curves, less clearly interpretable, is illustrated in the right-hand panel (b) of Figure S6 (compound 31, replicate R1). As before, the trace in well A11 was obtained in the absence of inhibitor; wells A5 - A8 correspond to inhibitor concentrations $317,100,32$, and $10 \mathrm{nM}$, respectively.

In this particular case, all reaction progress curves are again somewhat nonlinear, but it is no longer possible to conclude - simply upon visual examination - whether the observed nonlinearity is due to "slow-binding" inhibition or merely due to the slight curvature that is also seen in the substrate-only $([\mathrm{I}]=0)$ control curve. In fact, most reaction progress curves we observed belong into this second category.

\subsection{Theoretical models}

The experimental data from inhibition assays, exemplified in Figure S6, were subjected to global nonlinear regression analysis. In this section we describe the general form of the regression equation; three alternate mechanistic models and the corresponding systems of differential equations; two alternate treatment of fixed vs. adjustable model parameters; and a model-discrimination algorithm that was utilized to select the most plausible candidate kinetic mechanism for each particular inhibitor.

\subsubsection{The regression equation}

All progress curves arising from a given dose-response experiment, including the control progress curves obtained at zero inhibitor concentration, were combined and fit to Eqn (S1). However, the concentrations of reacting species $c_{i}(t)$ were computed by solving a system of firstorder ordinary differential equations (ODE) corresponding to one of several reaction mechanisms discussed below. Each ODE system was automatically derived by the DynaFit software package [4].

In order to properly characterize a variety of distinct features we identified in the overall shape of the reaction progress curves (see Figure S6), we have constructed three alternate regression models for each globally combined set of enzymatic progress curves.

4.2.2. Model R1S: "slow-binding”

$$
\begin{array}{rlllll}
\mathrm{E}+\mathrm{S} & \rightleftharpoons \mathrm{E} . \mathrm{S} & : & k_{\mathrm{a} . \mathrm{S}} & k_{\mathrm{d} . \mathrm{S}} \\
\mathrm{E} . \mathrm{S} & \rightarrow & \mathrm{E}+\mathrm{P} & : & k_{\mathrm{d} . \mathrm{P}} & \\
\mathrm{E}+\mathrm{P} & \rightleftharpoons & \mathrm{E} . \mathrm{P} & : & k_{\mathrm{a} . \mathrm{Pi}} & k_{\mathrm{d} . \mathrm{Pi}} \\
\mathrm{E} . \mathrm{S} & \rightarrow & \mathrm{F} & : & k_{\mathrm{ES}} & \\
\mathrm{E}+\mathrm{I} & \rightleftharpoons & \mathrm{E} . \mathrm{I} & : & k_{\mathrm{a} . \mathrm{I}} & k_{\text {d.I }}
\end{array}
$$

The first four steps in the above mechanism are exactly identical to model MM+pi+ed for SAM substrate kinetics (see section 1.2.3. The last step listed above describes a reversible binding of inhibitor I to the enzyme $\mathrm{E}$ to form the non-covalent enzyme-substrate complex E.I. The above mechanism is described mathematically by the ODE system of Eqns (S36)-(S43).

Importantly, when utilizing the above model R1S to fit the time-course of NNMT inhibition, both newly introduced rate constants, namely, $k_{\mathrm{a} . \mathrm{I}}$ that quantifies the enzyme-inhibitor association and $k_{\mathrm{d} . I}$ that describes the dissociation of the enzyme-inhibitor complex, are treated as adjustable model parameters. 


\subsubsection{Model R1F: "fast-binding"}

The molecular mechanism underlying this fitting model is exactly identical to the symbolic scheme shown in section 4.2.2 above. Thus, the corresponding system of differential equations is also exactly identical. The only important difference is that in model R1F we assumed that the intermolecular association between the enzyme and the inhibitor is instantaneous, or nearly instantaneous on the time scale of the experiment.

This "fast-binding" assumption was implemented by setting the bimolecular association rate constants $k_{\text {a.I }}$ to the arbitrarily chosen value of $10^{7} \mathrm{M}^{-1} \mathrm{~s}^{-1}$, or $10 \mu \mathrm{M}^{-1} \mathrm{~s}^{-1}$. This particular diffusion controlled value of $k_{\mathrm{a} . \mathrm{I}}$ was chosen on the basis well established precedents in the literature.[27, 28].

\subsubsection{Model C1: "irreversible" binding}

In a preliminary round of kinetic analyses (results not shown) we identified certain experimental data sets, for which the lower limit of the enzyme-inhibitor dissociation constant $k_{\mathrm{d} . I}$ could not be determined at the given confidence level, because it was essentially too low to measure reliably.

In order to assign an acceptable theoretical model to such exceptional data sets, we have included in our statistical model discrimination analysis the irreversible binding mechanism shown immediately below:

$$
\begin{aligned}
& \mathrm{E}+\mathrm{S} \rightleftharpoons \mathrm{E} . \mathrm{S} \quad: \quad \overrightarrow{k_{\mathrm{a} . \mathrm{S}}} \quad \overleftarrow{k_{\mathrm{d} . \mathrm{S}}} \\
& \text { E.S } \rightarrow \mathrm{E}+\mathrm{P} \quad: \quad k_{\text {d.P }} \\
& \mathrm{E}+\mathrm{P} \rightleftharpoons \mathrm{E} . \mathrm{P} \quad: \quad k_{\mathrm{a} . \mathrm{Pi}} k_{\mathrm{d} . \mathrm{Pi}} \\
& \mathrm{E} . \mathrm{S} \rightarrow \mathrm{F} \quad: \quad k_{\mathrm{ES}} \\
& \mathrm{E}+\mathrm{I} \rightarrow \text { E.I } \quad: \quad k_{\mathrm{a} . \mathrm{I}}
\end{aligned}
$$

According to this particular theoretical reaction scheme, the enzyme-inhibitor association is formally irreversible. However, this does not mean that the given inhibitor is being covalently attached to the enzyme target. Indeed it is reasonable to assume that all 35 inhibitors in our compound collection are reversible as opposed to covalent inhibitors. Instead, the true meaning and utility of model $\mathbf{C 1}$ is to express the fact that the lower limit of the confidence interval for the dissociation constant $k_{\mathrm{d} . \mathrm{I}}$ cannot be reached at the given confidence level.

\subsection{Two methods of handling substrate kinetic constants}

Our basic approach was to treat the substrate kinetic parameters for SAM as fixed parameters in the global analysis of the inhibition data. This treatment would ideally involve all floating substrate rate constants $k_{\mathrm{d} . \mathrm{S}}, k_{\mathrm{d} . \mathrm{P}}, k_{\mathrm{d} . \mathrm{Pi}}$ and $k_{\mathrm{ES}}$ that appear in the theoretical model $\mathbf{M M + p i + e d}$ described in section 1.2.4.

However, the complete collection of inhibition data consists of 105 plate-reader data sets obtained over an approximately six month period. Such extended period of time suggests that the kinetic properties of the enzyme might have changed, however subtly, over the course of time. For this reason, in the global fit of inhibition data we employed two different methods of analysis. 


\subsubsection{Method A: Fixed " $k_{\text {cat }}$ "}

According to this method, all substrate-related rate constants enumerated above, including in particular the " $k_{\mathrm{cat}}$ " equivalent labeled $k_{\mathrm{d} . \mathrm{P}}$ in the mechanistic scheme, were treated as fixed model parameters. The only adjustable rate constants were those related to inhibitor association and/or dissociation.

Additionally, in this method of analysis, we chose to also optimize the molar response coefficient of the fluorogenic product, $r_{\mathrm{P}}$ in Eqn (S1). This choice was necessitated by the fact that the kinetics work was performed on two different plate-reader instruments, which had distinct sensitivity characteristics. ${ }^{4}$ Finally, the list of adjustable model parameters included all baseline offsets on the signal axis, $F_{0}$ in Eqn (S1).

\subsubsection{Method B: Adjustable " $k_{\mathrm{cat}}$ "}

According to this alternate method, all substrate-related rate constants enumerated above, except the " $k_{\text {cat }}$ " equivalent labeled $k_{\mathrm{d} . \mathrm{P}}$ in the mechanistic scheme, were treated as fixed model parameters. Also treated as adjustable in the regression Eqn (S1) were rate constants pertaining to inhibitor association and/or dissociation.

The auxiliary model parameters $r_{\mathrm{P}}$, i.e., the molar responses coefficient of the reaction product, and $F_{0}$, i.e., the offsets on the signal axis, were treated in the same way as in Method A described above.

\section{Inhibition kinetics: Results}

In this section we first present representative examples of global fit and model discrimination for three types of kinetic behavior encountered in this study:

1. Inhibitors that display "fast" or instantaneous binding on the time-scale of the experiment.

2. Inhibitors that display prominent "slow" binding, or time dependence, clearly discernible even by simple visual examination of raw kinetic data.

3. Kinetic experiments that revealed that there is a "slow" component to enzyme-inhibitor interactions only upon close scrutiny.

We also report potency ranking of NS1 analogs obtained by two alternate data-analysis methods.

\subsection{Example 1: Compound 18, "fast-binding"}

Compound 18 was identified as one of the few compounds in this collection of 35 NNMT inhibitors that show very clear preference for the "fast-binding" inhibition mechanism R1F. This is illustrated in Figure S7. The inhibitor concentrations in well A2 through A10 were 100, 31.6, $10,3.16,1,0.316,0.1,0.0316$, and $0.01 \mu \mathrm{M}$, respectively. The experimental traces marked as A11 and A12 represent the negative control wells, where the inhibitor was absent.

The left-hand panel (a) in Figure $S 7$ shows the overlay of the experimental data points (various symbols) and the best-fit theoretical model curves, which were generated from the ODE

4 The two plate-reader instruments were the same model, from the same manufacturer, but displayed different sensitivity because of subtle mechanical and/or optical variation. 


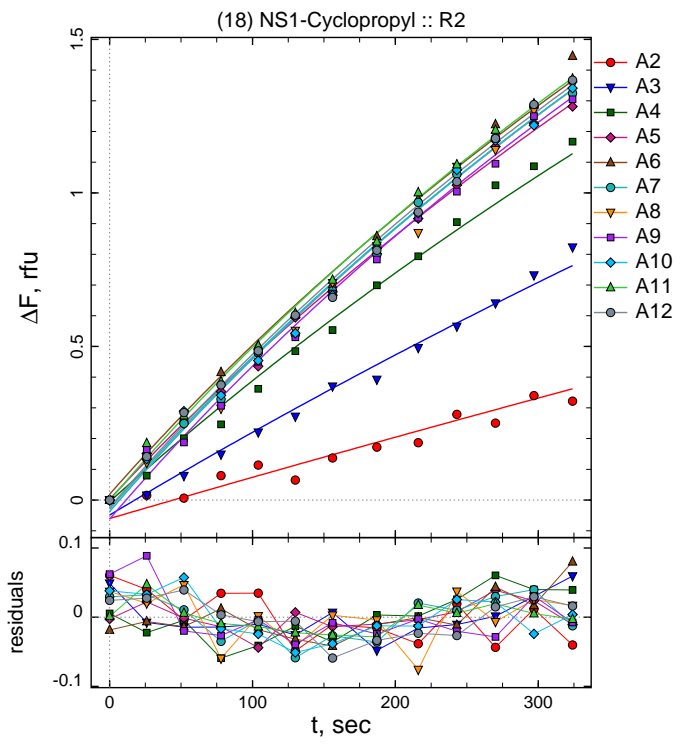

(a)

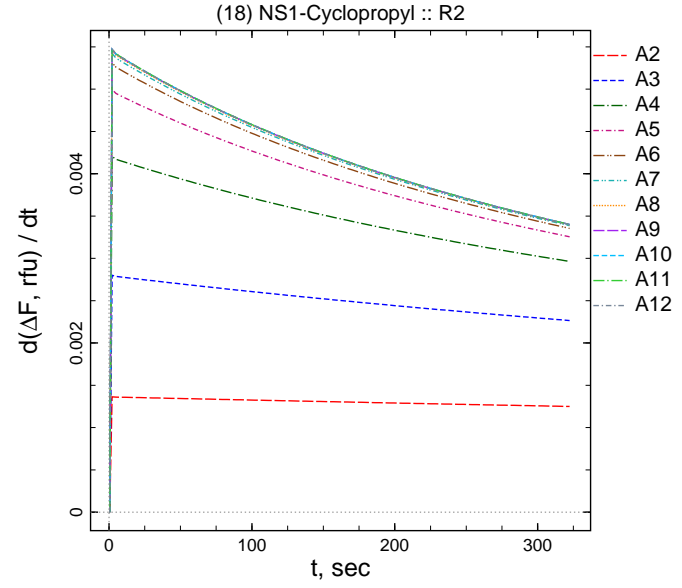

(b)

Figure S7: Global nonlinear least-squares fit of combined control progress curves from the inihibition assay of compound 18. For details see text.

system listed in Appendix B.2.1. The right-hand panel (b) displays the plot of instantaneous reaction rates, i.e., derivatives of the experimental model curves shown in panel (a) with respect to time. Note that the the reaction rate at time zero varies prominently with the inhibitor concentration. Such strong dependence of initial rates on the inhibitor concentration is a kinetic signature of the "fast-binding" mechanism R1F.

\subsubsection{Model discrimination analysis}

With regard to model discrimination analysis in the case of compound 18, as a typical example of "fast-binding" inhibitors in this compound collection, the detailed results auto-generated by the DynaFit software are listed in Appendix C.2. The two most salient observations that emerge on the basis of the detailed results are as follows:

1. The "fast-binding" kinetic model R1F and the "slow-binding" model R1S produced exactly identical residual sums of squares. However, model R1F is by definition associated with fewer adjustable parameters (i.e., the dissociation rate constant $k_{\text {d.I }}$ only) in comparison with model R1S (involving two adjustable rate constant, $k_{\mathrm{a} . \mathrm{I}}$ and $k_{\mathrm{d} . \mathrm{I}}$ ).

2. The confidence intervals for $k_{\mathrm{a} . \mathrm{I}}$ and $k_{\mathrm{d} . \mathrm{I}}$ in the "slow-binding" model R1S were halfopened from above as determined by the profile- $t$ method of Bates and Watts [8-10] $\left(\Delta \mathrm{SSQ}_{\mathrm{r}}=1 \%\right.$ according to the empirical method proposed by Johnson [14-16]). This is illustrated in Figure $S 8$.

Either of the first two observations listed above would be sufficient to exclude the "slowbinding" model R1S in favor of the "fast-binding" model R1F. However, the confidence interval 


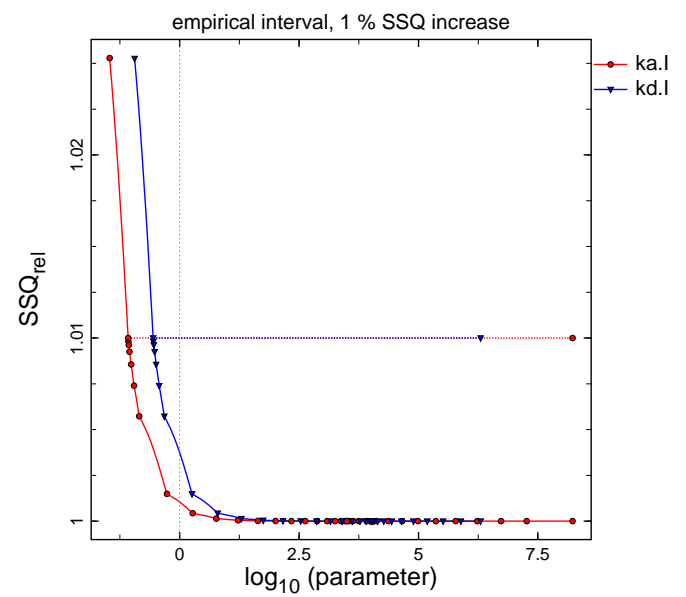

(a)

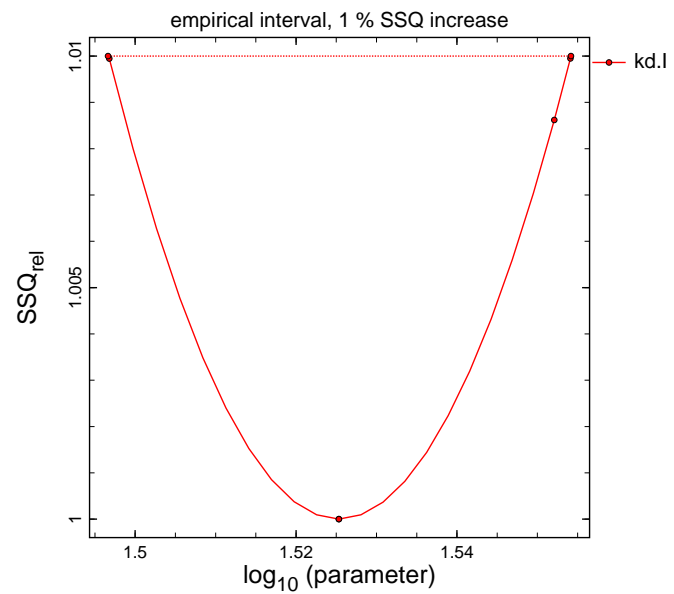

(b)

Figure S8: Confidence interval profiles for rate constants determined by the profile- $t$ method of Bates and Watts [8-10] in the fit of compound $\mathbf{1 8}$ inhibition data. (a) "Slow-binding" model R1S; (b) "fast-binding" model R1F.

profiles shown in Figure $S 8$ are particularly informative. Note in the left-hand panel (a) of Figure $S 8$ that the confidence interval profiles for either $k_{\mathrm{a} . \mathrm{I}}$ or $k_{\mathrm{d} . \mathrm{I}}$ display not even the slightest hint of a minimum on the least-squares hyper-surface. Only the lower limits for both rate constants could be determined at the $\Delta \mathrm{SSQ}_{\mathrm{r}}=1 \%$ confidence level.

\subsubsection{Model-invariance of the inhibition constant}

Another important observation that emerges from examination of the detailed model-selection results listed in Appendix C.2 is that the the best-fit values of the inhibition constant, defined as the ratio of the dissociation and association rate constants $K_{\mathrm{i}} \equiv k_{\mathrm{d} . \mathrm{I}} / k_{\mathrm{a} . \mathrm{I}}$, is invariant with respect to the choice of the fitting model. In other words, no matter which fitting model is considered as the "true" mechanism, either the fast-binding mechanism R1F or the slow-binding mechanism R1S, the inhibition constant comes out the same.

Note in the table labeled Intermediate results: Kinetic constants in Appendix C.2 that the best-fit values of the association and dissociation rate constants associated with the "slow-binding" model $\mathbf{R} 1$ are $k_{\mathrm{a} . \mathrm{I}}=5.35 \times 10^{6} \mu \mathrm{M}^{-1} \mathrm{~s}^{-1}$ and $k_{\mathrm{d} . \mathrm{I}}=1.8 \times 10^{7} \mathrm{~s}^{-1}$. The associated formal standard errors in the StdErr column are extremely large, corresponding to the coefficient of variation ( $C V$ column) greater than $5000 \%$. Thus, these particular "best-fit" values of $k_{\text {a.I }}$ and $k_{\text {d.I }}$ should be ignored as invalid.

However, also note that the ratio of the two (individually invalid) rate constants, $K_{\mathrm{i}} \equiv k_{\mathrm{d} . \mathrm{I}} / k_{\mathrm{a} . \mathrm{I}}=$ $1.8 \times 10^{7} / 5.35 \times 10^{6}=3.35 \mu \mathrm{M}$, is exactly identical to the equivalent value of $K_{\mathrm{i}}$ determined from "fast-binding" kinetic model R1F.

In particular, the third row of the Kinetic constants table in Appendix C.2 lists $k_{\mathrm{d} . \mathrm{I}}=33.5 \times$ $10^{7} \mathrm{~s}^{-1}$ as the best-fit value within model R1F. Recall that, according to the assumptions inherent in this particular kinetic model, the assumed (fixed) value of the enzyme-inhibitor association rate constant is $k_{\mathrm{a} . \mathrm{I}}=10 \mu \mathrm{M}^{-1} \mathrm{~s}^{-1}$. Thus, the "fast-binding" model results in $K_{\mathrm{i}} \equiv k_{\mathrm{d} . \mathrm{I}} / k_{\mathrm{a} . \mathrm{I}}=$ 
$33.5 / 10=3.35 \mu \mathrm{M}$, which is exactly the same result that would be obtained from the "slowbinding" model R1S.

\subsection{Example 2: Compound 26, "slow-binding"}

Compound 26 is an example of several compounds in this collection that displayed clear "slow-binding" behavior easily detectable even by simple visual inspection. This is illustrated in Figure S9. The inhibitor concentrations in well A5 through A10 were 130, 87, 58, 39, 26, and $17 \mathrm{nM}$, respectively. The enzyme concentration was $33 \mathrm{nM}$. The experimental traces marked as A11 and A12 represent the negative control wells, where the inhibitor was absent.

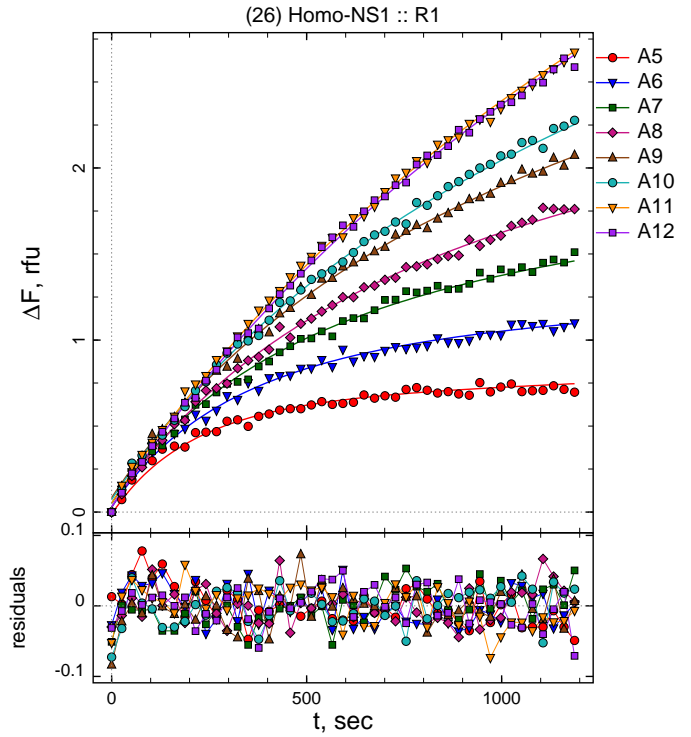

(a)

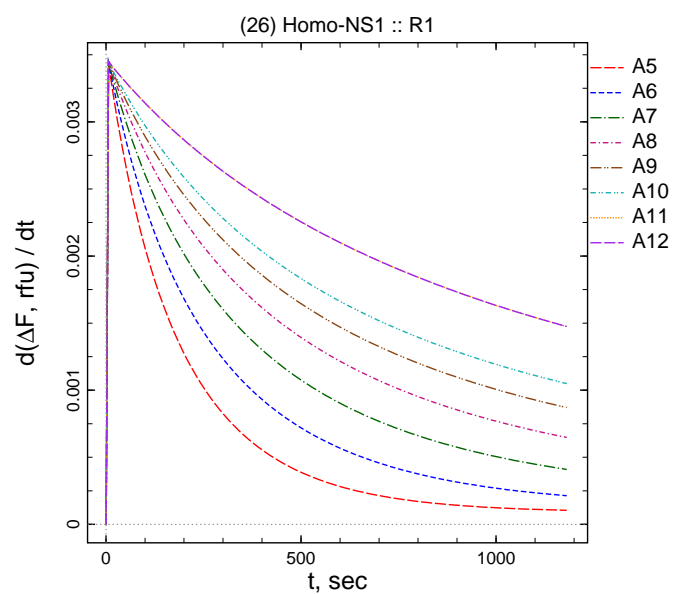

(b)

Figure S9: Global nonlinear least-squares fit of combined control progress curves from the inhibition assay of compound $\mathbf{2 6}$, replicate R1. For details see text.

The best-fit model curves displayed in Figure $S 9$ were generated by using Method A, in which the " $k_{\text {cat }}$ " rate constant $k_{\text {d.P }}$ was fixed at the best-fit value determined in the analysis of SAM kinetic data as described in Section 1. Nevertheless, as can be seen from the left-hand panel (a) of Figure S9, the negative control progress curves (wells A11 and A12) shows nearly perfect agreement with the presumed substrate kinetic properties.

This agreement (a) theoretical model curves based on the assumed (fixed) values of substraterelated rate constants and (b) the experimental traces observed in the absence of inhibitor is especially remarkable, given that the substrate-alone progress curves (wells A11 and A12) are strongly nonlinear. This is illustrated by the fact that the instantaneous reaction rate observed at $[\mathrm{I}]_{0}=0$, in the right-hand panel of Figure $S 9$, decreased by approximately $50 \%$ over the course of the 20-minute assay. 


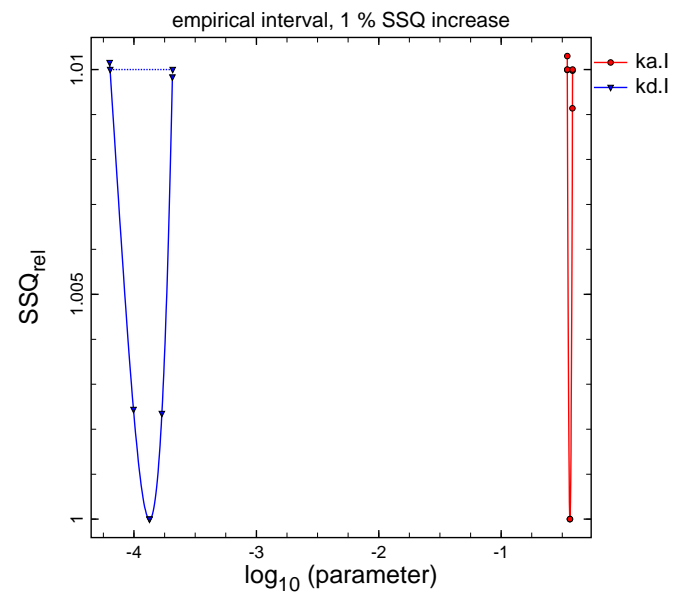

Replicate 1

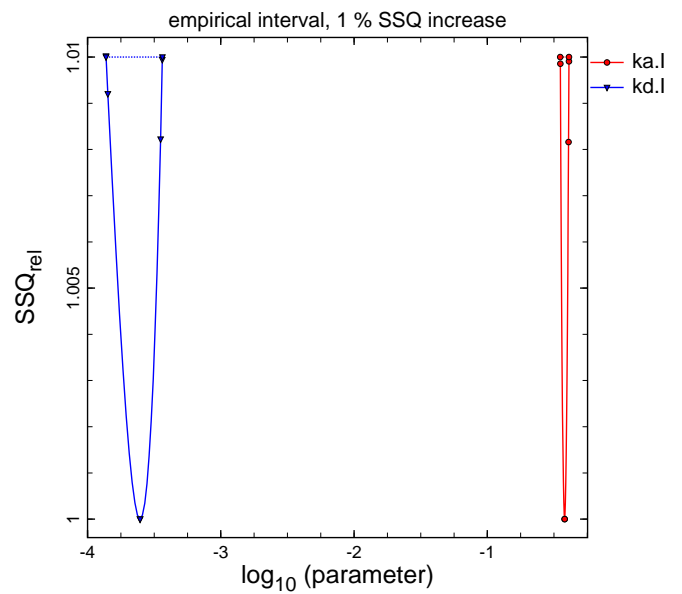

Replicate 2

Figure S10: Confidence interval profiles for rate constants determined by the profile- $t$ method of Bates and Watts [8-10] in the fit of compound 26 inhibition data.

\subsubsection{Model discrimination analysis}

With regard to model discrimination analysis in the case of compound 26, as a typical example of "slow-binding" inhibitors in this compound collection, the detailed results auto-generated by the DynaFit software are listed in Appendix C.3. The most salient observations that emerge on the basis of the detailed results are as follows:

1. Table Intermediate Results, Kinetic constants in Appendix C.3: All three candidate fitting models ("slow-binding" R1S, "fast-binding" R1F, and "irreversible" C1) resulted in the confidence intervals for all relevant rate constants being closed from both above and below. Thus, on the basis of confidence intervals alone, it is not possible to decide in favor of either model.

2. Table Final results, Information-theoretic criteria in Appendix C.3: The "fast-binding" model R1F can be excluded on the basis of the fact that it is associated with much higher residual sum of squares $\left(S S Q_{\mathrm{r}} \approx 7.475\right)$ relative to the "irreversible" model $\left(S S Q_{\mathrm{r}} \approx 1.037\right)$ even though both models contain the same number of adjustable parameters $\left(n_{\mathrm{P}}=10\right)^{5}$. However the "irreversible" binding model C1 cannot be excluded from consideration because the relative sum of squares is only $3.7 \%$ higher than the slow, reversible binding model R1S. This value is lower than the $5 \% \Delta S S Q_{\mathrm{r}}$ cut-off we required for a model to be formally disqualified.

Thus, in the absence of any other information, the "slow-binding" and "irreversible" binding models would both remain equally plausible, although the reversible model R1S is nominally favored because it has a slightly lower residual sum of squares, by approximately $4 \%$.

\footnotetext{
${ }^{5}$ The 10 adjustable model parameters for models R1F and $\mathbf{C 1}$ consist of one rate constant, one globally optimized molar response coefficient, and eight locally optimized offsets on the signal axis (one per progress curve).
} 
However, further support for the reversible "slow-binding" model R1S as opposed to the "irreversible" model $\mathbf{C 1}$ comes from two different sources. First, the chemical structure of compound 26 does not appear to support the idea that the compound could bind covalently to the enzyme. Second, the preference for model R1S has been observed in all three independently replicated experiments.

\subsubsection{Confidence intervals for inhibition rate constants}

Also very well reproduced across replicates R1-R3 is the fact the the confidence intervals or inhibition rate constants $k_{\mathrm{a} . \mathrm{I}}$ (association of the enzyme and inhibitor) and $k_{\mathrm{d} . \mathrm{I}}$ (dissociation of the noncovalent enzyme-inhibitor complex) are closed from both above and below. This is illustrated in Figure S10.

Note in Figure S10 that the best-fit values of the on-constant $k_{\text {a.I }}$ are particularly well replicated, as is indicated by the position of the minimum for $k_{\mathrm{a} . I}$ on the least-squares hyper-surface. The best-fit values of the off-constant $k_{\text {d.I }}$ are less well replicated and differ approximately twofold going from replicate R1 to replicate R3. Results for replicate R3 (not shown) were similar.

\subsubsection{Distribution of enzyme species}

The DynaFit software package [4] allows us to represent visually the distribution of various reacting species along the time-course of the enzymatic assay. In this case, this was arranged by inserting the following line of text into the appropriate DynaFit script:

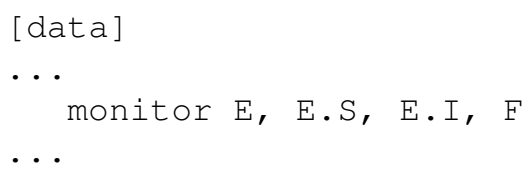

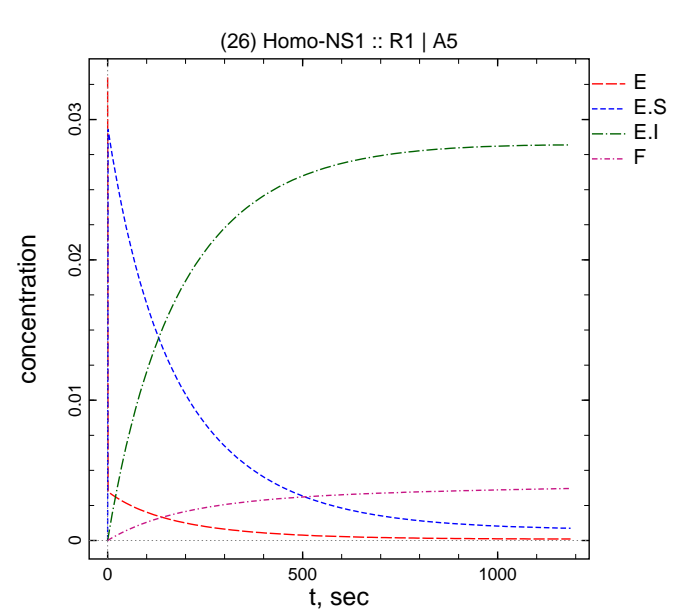

$[\mathrm{I}]_{0}=130 \mathrm{nM}$

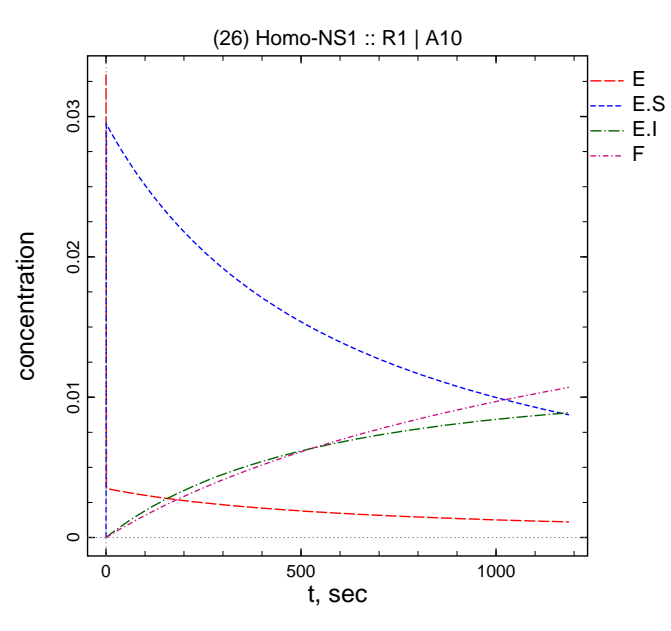

$[\mathrm{I}]_{0}=17 \mathrm{nM}$

Figure S11: Distribution of enzyme species in the inhibition assay of compound 26 at two different concentrations of the inhibitor. The enzyme concentration was $33 \mathrm{~nm}$. 
The results of this monitoring are displayed in Figure S11. According to model R1S and also using the assumed values of substrate-related rate constants determined in section 1 , the distribution curves shown in Figure S11 suggest that the enzyme-inhibitor complex is fully formed only at reaction times higher than approximately 10 minutes.

These and similar observations regarding the distribution of enzyme species should inform a thoughtful optimal design of kinetic experiments for the study of time-dependent enzyme inhibition, in particular with regard to the overall duration of the assay (in this case, at least 10-15 minutes).

\subsection{Example 3: Compound $\mathbf{3 1}$}

The enzyme-kinetic results for compound $\mathbf{3 1}$ are typical for the majority of progress curves obtained in this study, in several respects:

1. The duration of the assay was only 5 minutes, as opposed to the more optimal 15 to 20 minutes.

2. The model discrimination analysis resulted in both the "slow-binding" and the "fast-binding" models being given nearly identical weight.

3. However, in all three replicates "slow-binding" model slightly dominated as measured by the residual sum of squares, $S S Q_{\mathrm{r}}$.

4. Most importantly, the value of the inhibition constants defined as the ratio of rate constants $K_{\mathrm{i}} \equiv k_{\text {d.I }} / k_{\text {a.I }}$ was virtually insensitive to which model ("fast" or "slow") was assumed to be operative.

In the case of compound 31, and also most other compounds investigated in this study, it is quite challenging for a casual observer to detect that there is indeed a "slow-binding" component. This is illustrated in Figure S12. The inhibitor concentrations in well A5 through A10 were 316, $100,36,10,3.6$, and $1.0 \mathrm{nM}$, respectively. The nominal enzyme concentration was $100 \mathrm{nM}$. The experimental traces marked as A11 and A12 represent the negative control wells, where the inhibitor was absent.

Note in the right-hand panel (b) of Figure S12 that it takes approximately 60 and 90 seconds for the enzyme-inhibitor complex to be fully formed. This fact is most clearly visible in the case of progress curve labeled A6 corresponding to $100 \mathrm{nM}$ inhibitor. Only an experienced data analyst would probably detect the presence of this kinetic transient by simple visual examination of the raw data displayed in panel (a). However, most importantly, the slight transient feature is very well reproduced across all three independently replicated experiments.

\subsubsection{Model discrimination analysis}

With regard to model discrimination analysis in the case of compound $\mathbf{3 1}$, the detailed results auto-generated by the DynaFit software are listed in Appendix C.4. The most salient observations that emerge on the basis of the detailed results are as follows:

1. The inhibition constant defined as the ratio $K_{\mathrm{i}} \equiv k_{\mathrm{d} . \mathrm{I}} / k_{\mathrm{a} . \mathrm{I}}$ is largely invariant with respect to the presumed kinetic model ("slow" R1S or "fast" R1F).

2. The inhibition constant defined as the ratio $K_{\mathrm{i}} \equiv k_{\mathrm{d} . \mathrm{I}} / k_{\mathrm{a} . \mathrm{I}}$ is also very well reproduced across the three independently replicated experiments. Both of the above observations are documented in Table $S 2$ below. 


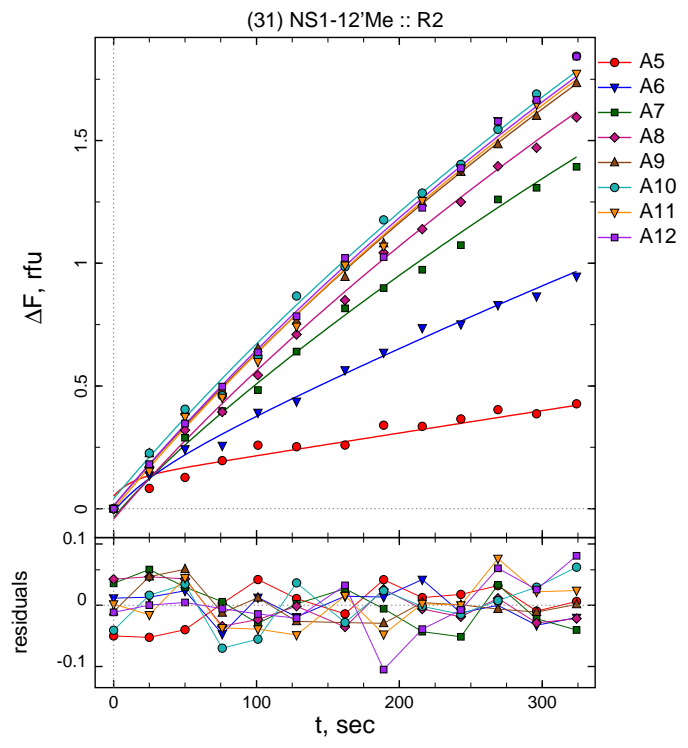

(a)

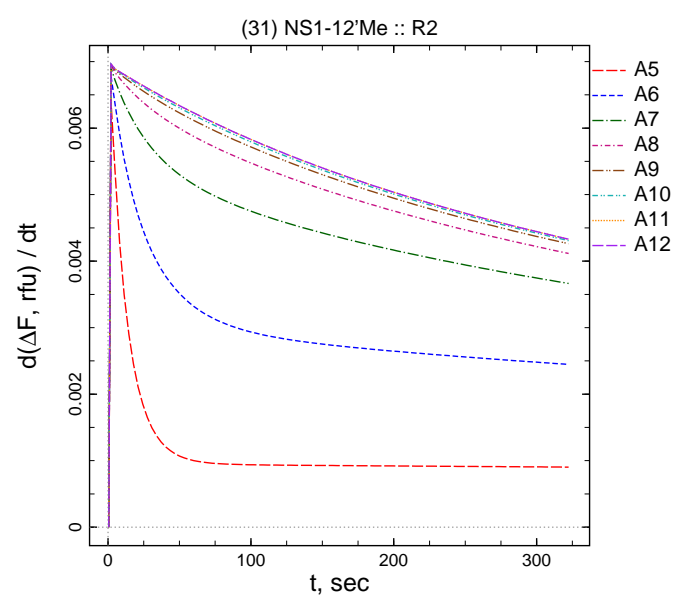

(b)

Figure S12: Global nonlinear least-squares fit of combined control progress curves from the inhibition assay of compound 31, replicate R2. For details see text.

\begin{tabular}{lccc}
\hline & \multicolumn{3}{c}{$K_{\mathrm{i}} \equiv k_{\mathrm{d} . \mathrm{I}} / k_{\mathrm{a} . \mathrm{I}}, \mathrm{nM}$} \\
model & replicate 1 & replicate 2 & replicate 3 \\
\hline "slow-binding" R1S & 3.3 & 3.8 & 2.5 \\
"fast-binding" R1F & 3.5 & 4.1 & 2.7 \\
\hline
\end{tabular}

Table S2: Inhibition constants for compound $\mathbf{3 1}$ under the assumption of "slow" or "fast" binding, across three independent replicates.

\subsubsection{Reproducibility and model-invariance of inhibition constants}

The $K_{\mathrm{i}}$ values listed in Table $S 2$ were compiled from DynaFit-generated tables labeled Intermediate results: Kinetic constants in Appendix C.4. For example, the value $K_{\mathrm{i}}=3.3 \mathrm{nM}$ for model R1S, replicate 1 , was computed as the ratio of $k_{\mathrm{d} . \mathrm{I}} / k_{\mathrm{a} . \mathrm{I}}=0.0168 / 4.96=0.0033 \mu \mathrm{M}=3.3$ nM. Similarly, the value $K_{\mathrm{i}}=3.5 \mathrm{nM}$ for model $\mathbf{R 1 F}$, replicate 1 , was computed as the ratio of $k_{\text {d.I }} / k_{\text {a.I }}=0.0348 / 10=0.00348 \mu \mathrm{M}=3.48 \mathrm{nM}$. Note that in the case of the "fast" binding model R1F we assumed that the enzyme-inhibitor association is described by the diffusion-controlled rate constant $k_{\mathrm{a} . \mathrm{I}}=10 \mu \mathrm{M}^{-1} \mathrm{~s}^{-1}=10^{7} \mathrm{M}^{-1} \mathrm{~s}^{-1}$. 


\subsection{Potency ranking of NS1 analogs}

The tables below summarize the potency ranking for NS1 analogs obtained by the two methods of analysis, namely, Method A (" $k_{\text {cat }}$ " rate constant fixed in the model) and Method B (" $k_{\text {cat }}$ " rate constant optimized). In table headings, $\mathrm{p} K_{\mathrm{i}}=-\log _{10} K_{\mathrm{i}}$ where $K_{\mathrm{i}}$ is in molar units (moles per liter).

\subsubsection{Method A}

\begin{tabular}{|c|c|c|c|c|c|c|}
\hline rank & NS1 analog & cpd. no. & $\mathrm{p} K_{\mathrm{i}} \mathrm{R} 1$ & $\mathrm{p} K_{\mathrm{i}} \mathrm{R} 2$ & $\mathrm{p} K_{\mathrm{i}} \mathrm{R} 3$ & aver. \pm sdev. \\
\hline 1 & Homo-NS1 & 26 & 9.90 & 9.62 & 10.00 & $9.84 \pm 0.20$ \\
\hline 2 & $\mathrm{NS} 1-12^{\prime} \mathrm{Cl}$ & 33 & 9.91 & 9.56 & 9.70 & $9.72 \pm 0.18$ \\
\hline 3 & NS1 & 10 & 9.27 & 9.47 & 9.42 & $9.38 \pm 0.11$ \\
\hline 4 & NS1-12'F & 30 & 8.66 & 8.89 & 8.72 & $8.76 \pm 0.12$ \\
\hline 5 & NS1-12'CF3 & 32 & 8.54 & 8.68 & 8.57 & $8.60 \pm 0.07$ \\
\hline 6 & $\mathrm{NS} 1-12^{\prime} \mathrm{Me}$ & 31 & 8.48 & 8.43 & 8.61 & $8.50 \pm 0.09$ \\
\hline 7 & NS1-Alkane & 15 & 8.37 & 8.37 & 8.46 & $8.40 \pm 0.05$ \\
\hline 8 & NS1-Pyr13' & 39 & 8.38 & 8.40 & 8.36 & $8.38 \pm 0.02$ \\
\hline 9 & NS1-Pyr10' & 37 & 8.15 & 8.21 & 8.28 & $8.21 \pm 0.07$ \\
\hline 10 & NS1-Pyr12' & 38 & 8.21 & 8.05 & 8.10 & $8.12 \pm 0.08$ \\
\hline 11 & NS1-AminoAmide & 24 & 7.84 & 7.88 & 7.85 & $7.86 \pm 0.02$ \\
\hline 12 & NS1-Methylenedioxy & 36 & 7.50 & 7.56 & 7.51 & $7.52 \pm 0.04$ \\
\hline 13 & NS1-Pyr14' & 40 & 7.43 & 7.48 & 7.52 & $7.48 \pm 0.04$ \\
\hline 14 & NS1-Benzolactam6 & 34 & 7.36 & 7.39 & 7.34 & $7.37 \pm 0.03$ \\
\hline 15 & NS1-Amine & 21 & 7.32 & 7.31 & 7.33 & $7.32 \pm 0.01$ \\
\hline 16 & & MS2734 & 7.15 & 7.13 & 7.16 & $7.15 \pm 0.01$ \\
\hline 17 & NS1-Urea & 25 & 7.17 & 7.09 & 7.13 & $7.13 \pm 0.04$ \\
\hline 18 & NS1-Alkane 6'Epi & 16 & 6.98 & 6.92 & 6.96 & $6.95 \pm 0.03$ \\
\hline 19 & NS1-6’Epi & 14 & 6.85 & 6.88 & 6.97 & $6.90 \pm 0.06$ \\
\hline 20 & NS1-Sulfonamide & 29 & 6.71 & 6.84 & 6.73 & $6.76 \pm 0.07$ \\
\hline 21 & Mini-NS1 & 17 & 6.63 & 6.59 & 6.56 & $6.59 \pm 0.04$ \\
\hline 22 & NS1-MethylEster & 23 & 6.44 & 6.32 & 6.35 & $6.37 \pm 0.06$ \\
\hline 23 & & MS2756 & 6.08 & 6.14 & 6.12 & $6.12 \pm 0.03$ \\
\hline 24 & NS1-Amide & 22 & 6.01 & 6.06 & 6.00 & $6.02 \pm 0.03$ \\
\hline 25 & NS1-Benzolactam5 & 35 & 5.79 & 5.77 & 5.83 & $5.79 \pm 0.03$ \\
\hline 26 & NS1-Cyclopropyl & 18 & 5.49 & 5.47 & 5.57 & $5.51 \pm 0.05$ \\
\hline 27 & NS1-Aminonaphthalene & 41 & 5.54 & 5.48 & 5.48 & $5.50 \pm 0.03$ \\
\hline 28 & NS1- $p$ Benzamide & 27 & 5.28 & 5.30 & 5.30 & $5.29 \pm 0.01$ \\
\hline 29 & & VH45 & 5.27 & 5.30 & 5.30 & $5.29 \pm 0.02$ \\
\hline 30 & NS1-Phenyl & 13 & 4.96 & 4.94 & 5.03 & $4.98 \pm 0.04$ \\
\hline 31 & NS1-oBenzamide & 28 & 4.81 & 4.77 & 4.79 & $4.79 \pm 0.02$ \\
\hline 32 & Desthia-SAH & 11 & 4.72 & 4.76 & 4.76 & $4.75 \pm 0.02$ \\
\hline 33 & NS1-Carboxylic Acid & 20 & 4.67 & 4.78 & 4.75 & $4.73 \pm 0.06$ \\
\hline 34 & NS1-Alkyne & 12 & 4.37 & 4.34 & 4.31 & $4.34 \pm 0.03$ \\
\hline 35 & NS1-Desadenine & 19 & 4.19 & 4.19 & 4.07 & $4.15 \pm 0.07$ \\
\hline
\end{tabular}


5.4.2. Method B

\begin{tabular}{|c|c|c|c|c|c|c|}
\hline rank & NS1 analog & cpd. no. & $\mathrm{p} K_{\mathrm{i}} \mathrm{R} 1$ & $\mathrm{p} K_{\mathrm{i}} \mathrm{R} 2$ & $\mathrm{p} K_{\mathrm{i}} \mathrm{R} 3$ & aver. \pm sdev. \\
\hline 1 & $\mathrm{NS} 1-12^{\prime} \mathrm{Cl}$ & 33 & 9.88 & 9.59 & 9.70 & $9.72 \pm 0.15$ \\
\hline 2 & Homo-NS1 & 26 & 9.68 & 9.57 & 9.63 & $9.62 \pm 0.05$ \\
\hline 3 & NS1 & 10 & 9.23 & 9.36 & 9.32 & $9.30 \pm 0.07$ \\
\hline 4 & NS1-12'F & 30 & 8.63 & 8.90 & 8.67 & $8.73 \pm 0.15$ \\
\hline 5 & NS1-12'CF3 & 32 & 8.51 & 8.68 & 8.55 & $8.58 \pm 0.08$ \\
\hline 6 & NS1-12’Me & 31 & 8.44 & 8.39 & 8.57 & $8.47 \pm 0.09$ \\
\hline 7 & NS1-Alkane & 15 & 8.31 & 8.32 & 8.36 & $8.33 \pm 0.03$ \\
\hline 8 & NS1-Pyr13' & 39 & 8.29 & 8.32 & 8.28 & $8.30 \pm 0.02$ \\
\hline 9 & NS1-Pyr10' & 37 & 8.11 & 8.19 & 8.22 & $8.17 \pm 0.05$ \\
\hline 10 & NS1-Pyr12' & 38 & 8.17 & 8.00 & 7.99 & $8.05 \pm 0.10$ \\
\hline 11 & NS1-AminoAmide & 24 & 7.74 & 7.78 & 7.79 & $7.77 \pm 0.02$ \\
\hline 12 & NS1-Methylenedioxy & 36 & 7.46 & 7.54 & 7.46 & $7.49 \pm 0.05$ \\
\hline 13 & NS1-Pyr14 & 40 & 7.38 & 7.45 & 7.51 & $7.45 \pm 0.07$ \\
\hline 14 & NS1-Benzolactam6 & 34 & 7.28 & 7.29 & 7.20 & $7.26 \pm 0.05$ \\
\hline 15 & NS1-Amine & 21 & 7.25 & 7.22 & 7.25 & $7.24 \pm 0.02$ \\
\hline 16 & NS1-Urea & 25 & 7.17 & 7.07 & 7.11 & $7.12 \pm 0.05$ \\
\hline 17 & & MS2734 & 7.05 & 7.04 & 7.06 & $7.05 \pm 0.01$ \\
\hline 18 & NS1-Alkane 6'Epi & 16 & 7.03 & 6.92 & 6.95 & $6.97 \pm 0.05$ \\
\hline 19 & NS1-6’Epi & 14 & 6.87 & 6.87 & 6.99 & $6.91 \pm 0.07$ \\
\hline 20 & NS1-Sulfonamide & 29 & 6.68 & 6.77 & 6.68 & $6.71 \pm 0.05$ \\
\hline 21 & Mini-NS1 & 17 & 6.62 & 6.59 & 6.53 & $6.58 \pm 0.04$ \\
\hline 22 & NS1-MethylEster & 23 & 6.46 & 6.31 & 6.32 & $6.36 \pm 0.08$ \\
\hline 23 & & MS2756 & 5.98 & 6.09 & 6.04 & $6.04 \pm 0.06$ \\
\hline 24 & NS1-Amide & 22 & 5.97 & 5.99 & 5.98 & $5.98 \pm 0.01$ \\
\hline 25 & NS1-Benzolactam5 & 35 & 5.73 & 5.71 & 5.75 & $5.73 \pm 0.02$ \\
\hline 26 & NS1-Aminonaphthalene & 41 & 5.52 & 5.40 & 5.38 & $5.43 \pm 0.07$ \\
\hline 27 & NS1-Cyclopropyl & 18 & 5.38 & 5.37 & 5.47 & $5.40 \pm 0.05$ \\
\hline 28 & NS1- $p$ Benzamide & 27 & 5.22 & 5.24 & 5.25 & $5.24 \pm 0.02$ \\
\hline 29 & & VH45 & 5.18 & 5.22 & 5.22 & $5.21 \pm 0.02$ \\
\hline 30 & NS1-Phenyl & 13 & 4.88 & 4.88 & 4.94 & $4.90 \pm 0.03$ \\
\hline 31 & NS1-oBenzamide & 28 & 4.71 & 4.69 & 4.72 & $4.71 \pm 0.02$ \\
\hline 32 & Desthia-SAH & 11 & 4.65 & 4.67 & 4.68 & $4.67 \pm 0.01$ \\
\hline 33 & NS1-Carboxylic Acid & 20 & 4.59 & 4.69 & 4.67 & $4.65 \pm 0.06$ \\
\hline 34 & NS1-Alkyne & 12 & 4.39 & 4.33 & 4.24 & $4.32 \pm 0.08$ \\
\hline 35 & NS1-Desadenine & 19 & 4.21 & 4.22 & 3.91 & $4.11 \pm 0.17$ \\
\hline
\end{tabular}

\subsubsection{Comparison of results}

A comparison of results contained in the two summary tables listed immediately above reveals that the ranking of NS1 inhibitors by potency does not change upon going from Method A (fixed " $k_{\text {cat }}$ ") to Method B (optimized " $k_{\text {cat }}$ ") - as long as the statistical uncertainty of $\mathrm{p} K_{\mathrm{i}}$ determinations is properly taken into account. This is true even though the nominal order of potency has changed from Method A to Method B in several cases. In particular, the "best" or most potent NS1 analog is compound 26 according to Method A, while at the same the "best" or most potent NS1 analog according to Method B is compound 33. However, within both methods the $\mathrm{p} K_{\mathrm{i}}$ values for both "top" ranked compounds are identical within the margin or error. 


\section{References}

[1] P. Kuzmič, A. G. Peranteau, G. Garcia-Echeverria, D. H. Rich, Mechanical effects on the kinetics of the HIV proteinase deactivation, Biochem. Biophys. Res. Commun. 221 (1996) 313-7.

[2] M. Sadana, Biocatalysis - Fundamentals of Enzyme Deactivation Kinetics, Prentice-Hall, Englewood Cliffs, NJ, 1991.

[3] P. Kuzmič, Program DYNAFIT for the analysis of enzyme kinetic data: Application to HIV proteinase, Anal. Biochem. 237 (1996) 260-273.

[4] P. Kuzmič, DynaFit - A software package for enzymology, Meth. Enzymol. 467 (2009) 247-280.

[5] J. M. Beechem, Global analysis of biochemical and biophysical data, Meth. Enzymol. 210 (1992) 37-54.

[6] H. Neelakantan, V. Vance, H. L. Wang, S. F. McHardy, S. J. Watowich, Noncoupled fluorescent assay for direct real-time monitoring of nicotinamide $\mathrm{N}$-methyltransferase activity, Biochemistry 56 (2017) 824-832.

[7] Y. Peng, D. Sartini, V. Pozzi, D. Wilk, M. Emanuelli, V. C. Yee, Structural basis of substrate recognition in human nicotinamide N-methyltransferase, Biochemistry 50 (2011) 7800 7808 .

[8] D. M. Bates, D. G. Watts, Nonlinear Regression Analysis and its Applications, Wiley, New York, 1988.

[9] D. G. Watts, Parameter estimation from nonlinear models, Meth. Enzymol. 240 (1994) 24-36.

[10] I. Brooks, D. G. Watts, K. K. Soneson, P. Hensley, Determining confidence intervals for parameters derived from analysis of equilibrium analytical ultracentrifugation data, Meth. Enzymol. 240 (1994) 459-78.

[11] K. B. Burnham, D. R. Anderson, Model Selection and Multimodel Inference: A Practical Information-Theoretic Approach, 2nd Edition, Springer-Verlag, New York, 2002.

[12] J. I. Myung, M. A. Pitt, Model comparison methods, Meth. Enzymol. 383 (2004) 351-366.

[13] J. I. Myung, Y. Tang, M. A. Pitt, Evaluation and comparison of computational models, Meth. Enzymol. 454 (2009) 287-304.

[14] K. A. Johnson, Z. B. Simpson, T. Blom, Global Kinetic Explorer: A new computer program for dynamic simulation and fitting of kinetic data, Anal. Biochem. 387 (2009) 20-29.

[15] K. A. Johnson, Z. B. Simpson, T. Blom, FitSpace Explorer: An algorithm to evaluate multidimensional parameter space in fitting kinetic data, Anal. Biochem. 387 (2009) 30-41.

[16] K. A. Johnson, Fitting enzyme kinetic data with KinTek Global Kinetic Explorer, Meth. Enzymol. 467 (2009) 601-626. 
[17] M. L. Reytor-Gonzalez, S. Cornell-Kennon, E. Schaffer, P. Kuzmič, An algebraic model for the determination of Michaelis-Menten kinetic parameters by global nonlinear fit of enzymatic progress curves, Anal. Biochem. 518 (2017) 16-24.

[18] W. W. Cleland, The kinetics of enzyme-catalyzed reactions with two or more substrates or products: I. Nomenclature and rate equations, Biochim. Biophys. Acta 67 (1963) 104-137.

[19] A. Cornish-Bowden, Fundamentals of Enzyme Kinetics, 4th Edition, Wiley-VCH Verlag GmbH \& Co. KGaA, Berlin, 2012.

[20] H. S. Loring, P. R. Thompson, Kinetic mechanism of nicotinamide N-nethyltransferase, Biochemistry 57 (2018) 5524-5532.

[21] J. W. Williams, J. F. Morrison, The kinetics of reversible tight-binding inhibition, Meth. Enzymol. 63 (1979) 437-467.

[22] S. Cha, Tight-binding inhibitors. I. Kinetic behavior, Biochem. Pharmacol. 24 (1975) 21772185.

[23] J. F. Morrison, Kinetics of the reversible inhibition of enzyme-catalysed reactions by tightbinding inhibitors, Biochim. Biophys. Acta 185 (1969) 269-286.

[24] K. B. Taylor, Enzyme Kinetics and Mechanisms, Kluwer Academic Publishing, Dordrecht, 2002.

[25] J. F. Morrison, C. T. Walsh, The behavior and significance of slow-binding enzyme inhibitors, Adv. Enzymol. Related Areas Mol. Biol. 61 (1988) 201-301.

[26] S. Szedlacsek, R. G. Duggleby, Kinetics of slow and tight-binding inhibitors, Meth. Enzymol. 249 (1995) 144-180.

[27] T. E. Creighton, Proteins: Structures and Molecular Properties, 2nd Edition, W. H. Freeman, San Francisco, 1992.

[28] A. Fersht, Structure and Mechanism in Protein Science: A Guide to Enzyme Catalysis and Protein Folding, 3rd Edition, W. H. Freeman, New York, 1999. 


\section{Appendix}

\section{A. DynaFit scripts}

This appendix contains the verbatim listing of example script input files for the software package DynaFit [4].

\section{A.1. Global fit of SAM substrate kinetic data}

The following DynaFit script file was used to fit one of the three replicates (in this case R1) of SAM substrate saturation data to the four candidate kinetic mechanisms displayed in sections 1.2.1-1.2.4, represented mathematically by the ODE system shown in Appendix B.1.1-B.1.4.

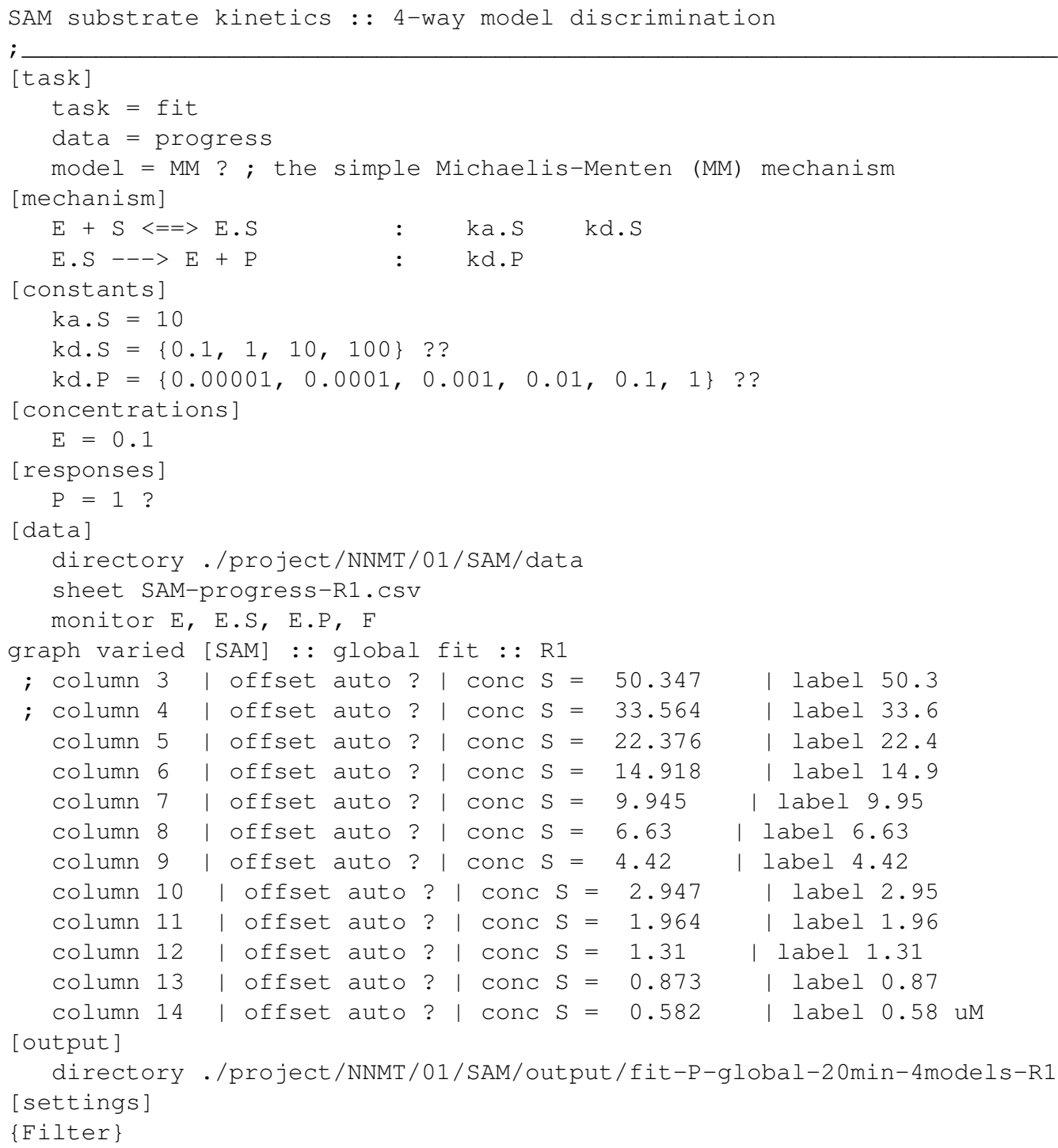




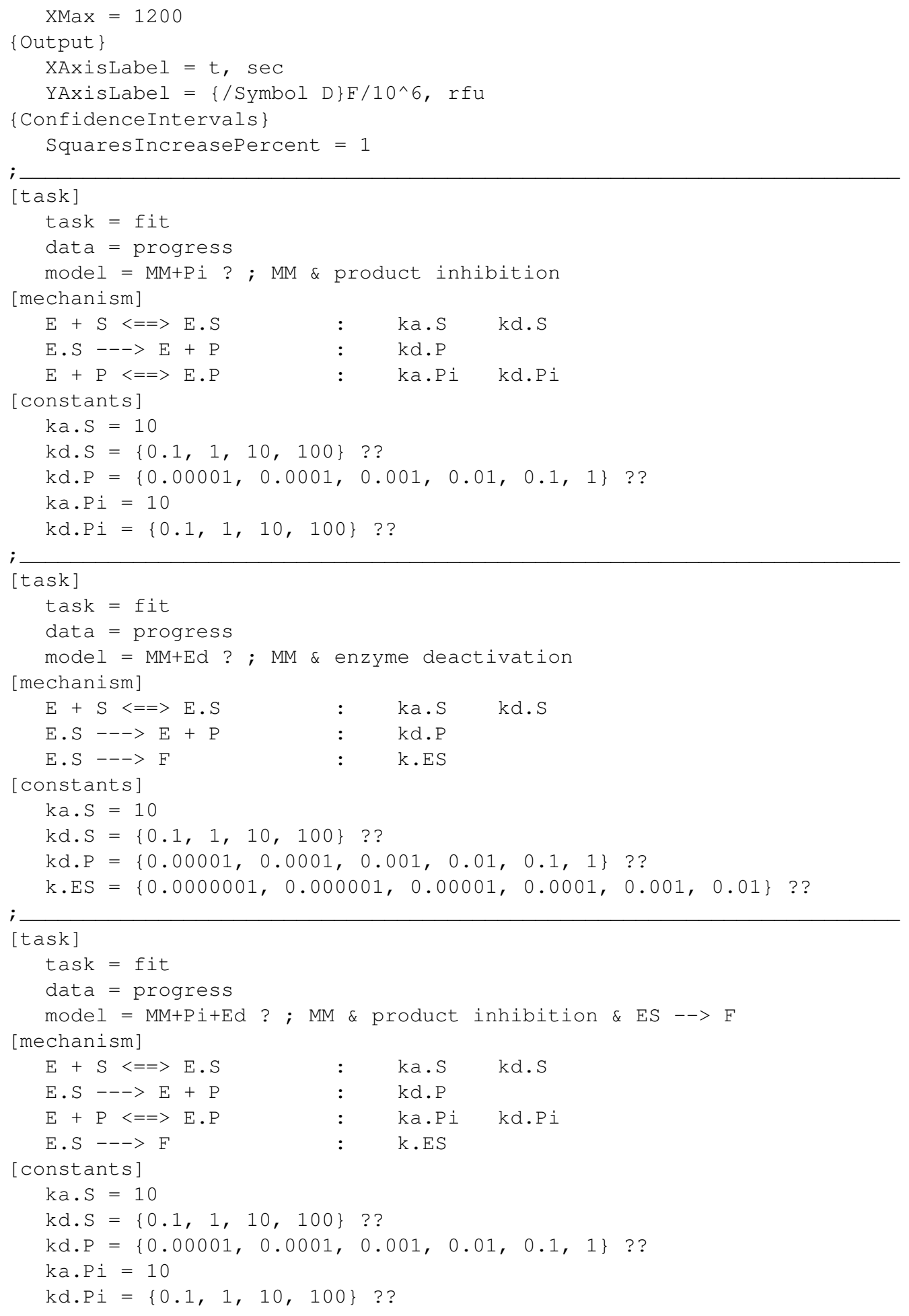



[end]

$\mathrm{k} . \mathrm{ES}=\{0.0000001,0.000001,0.00001,0.0001,0.001,0.01\}$ ?? ;

\section{A.2. Local fit of quinoline substrate kinetic data}

The following DynaFit script file was used to fit one of the three replicates (in this case R1) of quinoline substrate saturation data the integrated Michaelis-Menten rate equation, based on the Lambert Omega function [17], in order to determine the initial reaction rates.

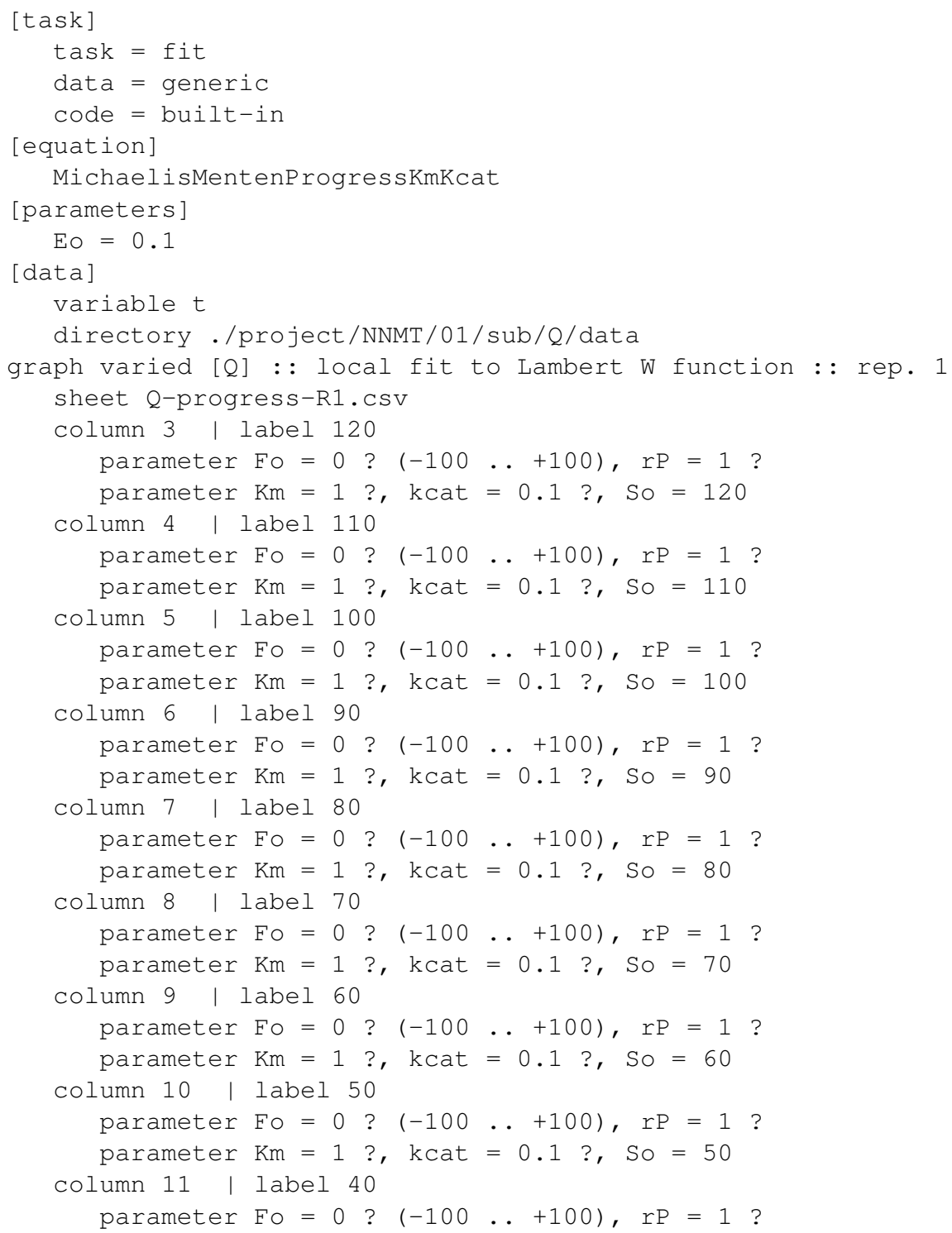




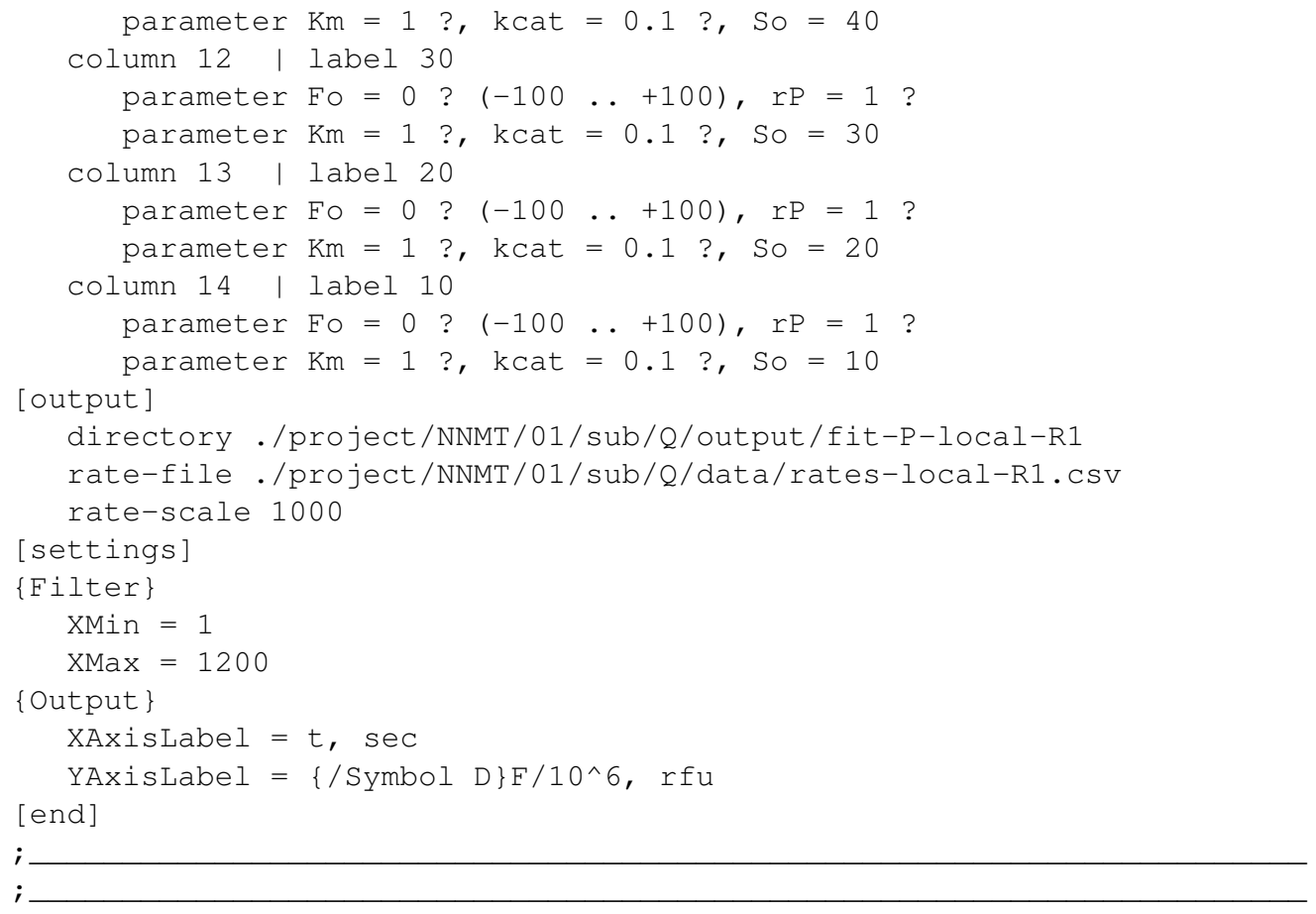

\section{A.3. Determination of the apparent Michaelis constant for quinoline}

The following DynaFit script file was used to fit initial reaction rates to determine the apparent Michaelis constant for quinoline. The reaction rates exported automatically the DynaFit script listed in Appendix A.2 were pooled into a single data file.

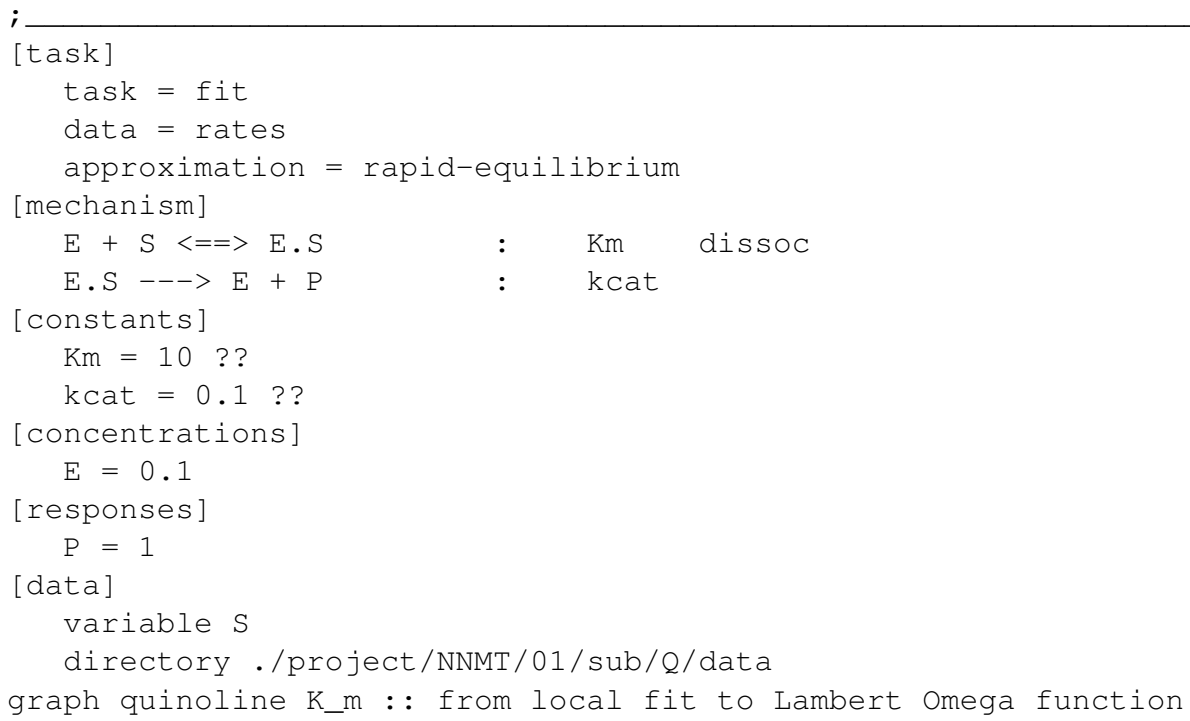


sheet rates-local-R123.csv

column 2

[output]

directory ./project/NNMT/01/sub/Q/output/fit-V-local-R123

[settings]

$\{$ Filter $\}$

AverageReplicates = y

\{Output\}

XAxisLabel $=$ [Quinoline,$\{/$ Symbol $\mathrm{m}\} \mathrm{M}$

YAxisLabel $=\mathrm{v} / 10^{\wedge} 3$, rfu/sec

PredictionBands $=\mathrm{y}$

[end]

i

A.4. Global fit of inhibition data, Cpd 31, Rep 1

The following DynaFit script file was used to fit one of the three replicates (in this case R1) of inhibition data involving compound $\mathbf{3 1}$ to the three candidate mechanisms displayed in section 4.2, which are represented mathematically by the ODE systems shown in Appendix B.2.

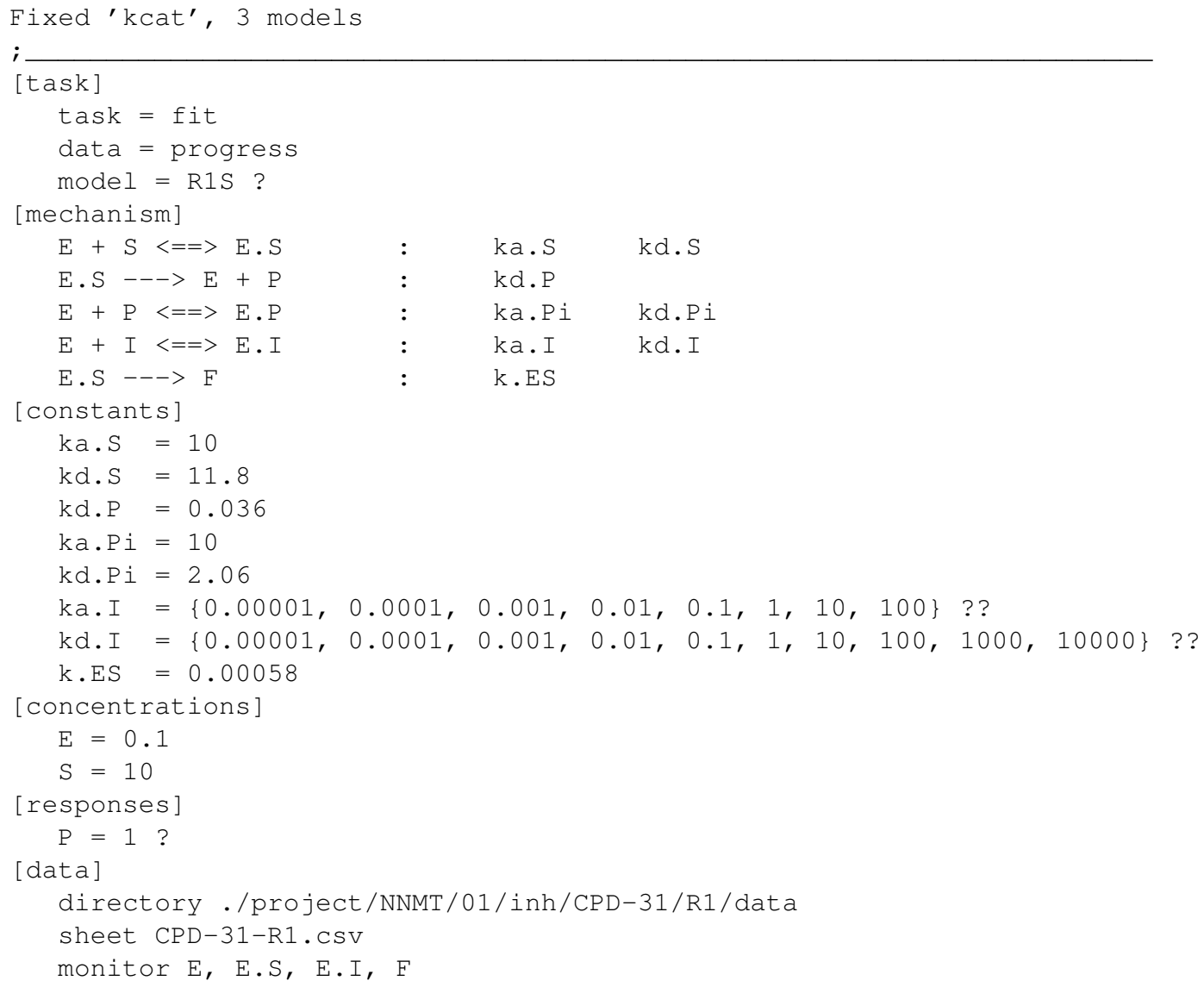




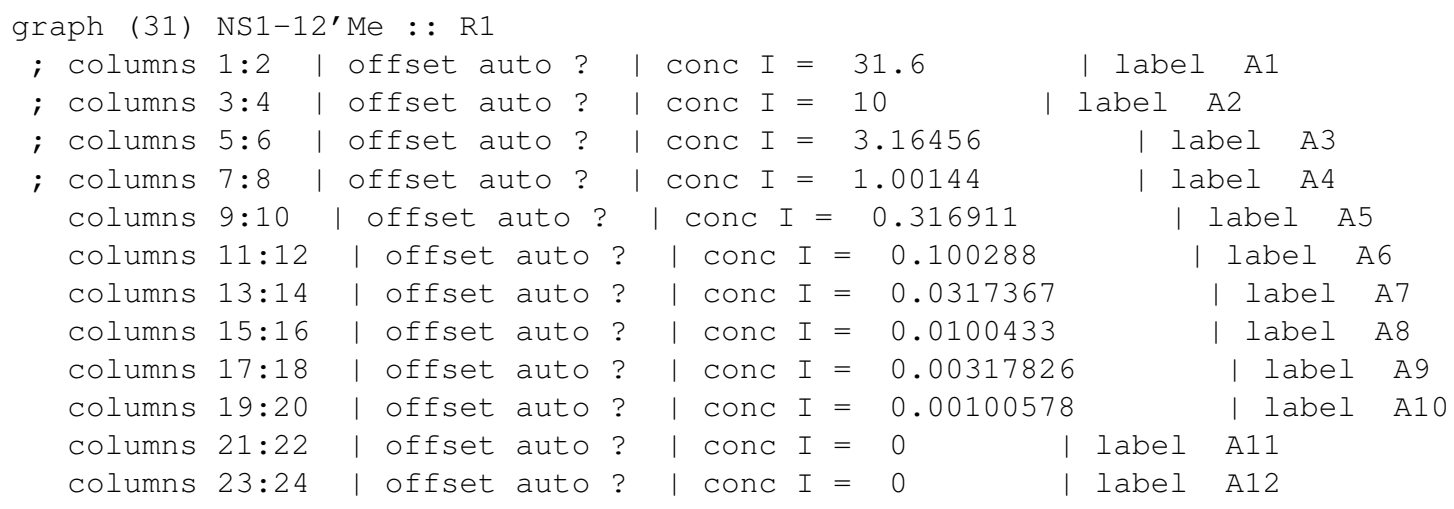

[output]

directory ./project/NNMT/01/inh/CPD-31/R1/output/fit-CPD-31-R1-001

[settings]

$\{$ Filter $\}$

$\mathrm{XMin}=0$

$\mathrm{XMax}=1200$

ZeroBaselineSignal $=y$

\{Output\}

XAxisLabel $=t$, sec

YAxisLabel $=\{/$ Symbol D $\}$, rfu

WriteTeX $=y$

\{Constraints\}

Concentrations $=1.414$

\{ConfidenceIntervals\}

SquaresIncreasePercent $=1$

Onlyconstants $=\mathrm{n}$

\{EstimateScan\}

RefineEstimates $=30$

\{Modelselection\}

Onlyconstants $=\mathrm{y}$

CoefficientofVariationMax $=1000000000000$

CoefficientofVariationSearchMax $=1000000000000$

task]

task $=$ fit

data $=$ progress

model $=$ R1F ?

[mechanism]

$\begin{array}{llll}\mathrm{E}+\mathrm{S}<==>\mathrm{E} . \mathrm{S} & : & \mathrm{ka} . \mathrm{S} & \mathrm{kd} . \mathrm{S} \\ \mathrm{E} . \mathrm{S}--->\mathrm{E}+\mathrm{P} & : & \mathrm{kd} . \mathrm{P} & \\ \mathrm{E}+\mathrm{P}<==>\mathrm{E} . \mathrm{P} & : & \mathrm{ka} . \mathrm{Pi} & \mathrm{kd} . \mathrm{Pi} \\ \mathrm{E}+\mathrm{I}<==>\mathrm{E} . \mathrm{I} & : & \mathrm{ka} . \mathrm{I} & \mathrm{kd} . \mathrm{I} \\ \mathrm{E} . \mathrm{S}-->\mathrm{F} & : & \mathrm{k} . \mathrm{E} \mathrm{S} & \end{array}$

[constants]

$\mathrm{ka} . \mathrm{S}=10$

$\mathrm{kd} . \mathrm{s}=11.8$

$\mathrm{kd} . \mathrm{P}=0.036$ 


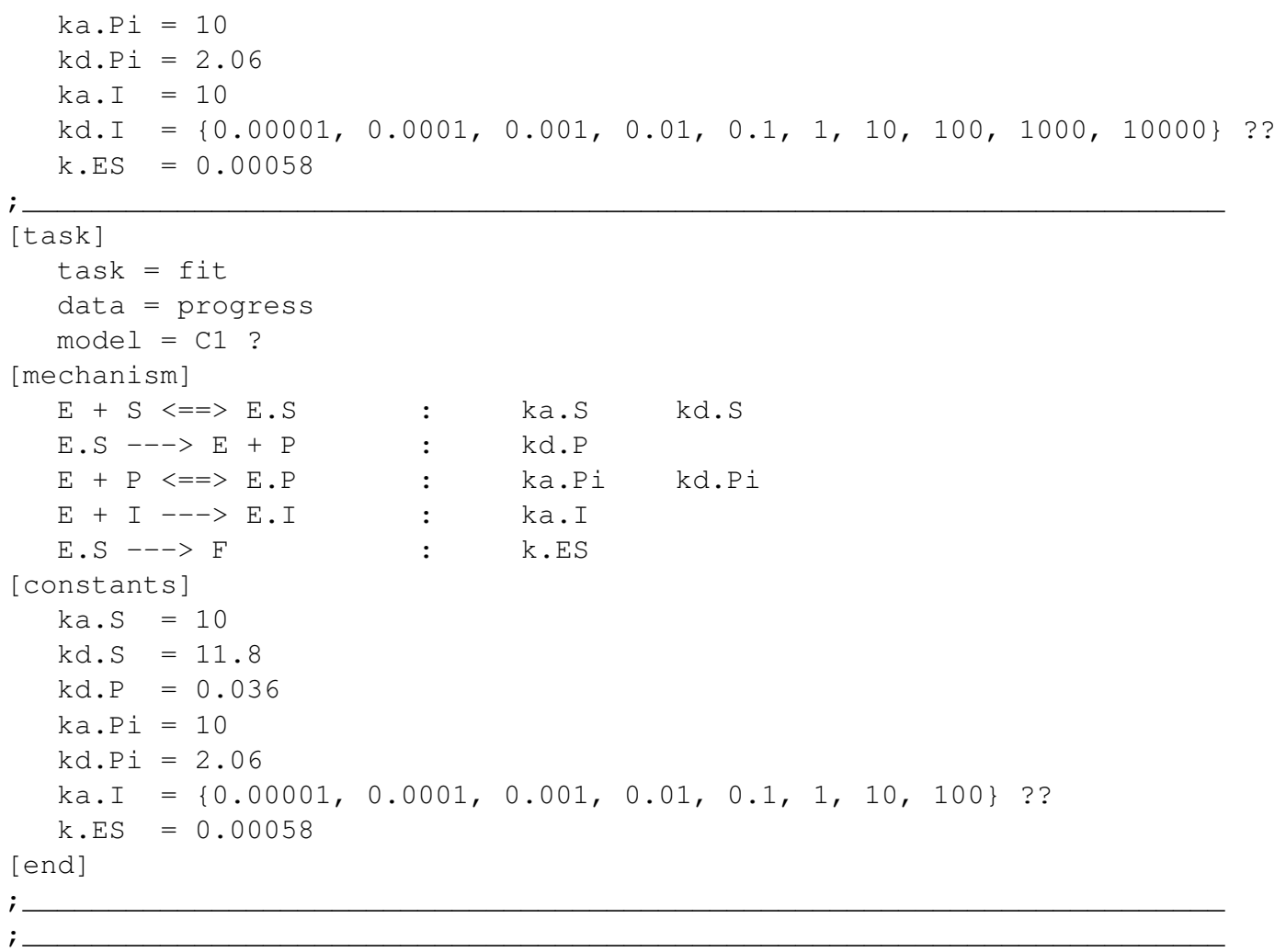

The DynaFit script listed above corresponds to Method A, where the " $k_{\text {cat }}$ " rate constant labeled $k_{\text {d.I }}$ is treated as a fixed parameter set to the value determined independently as described in section 1.3.2.

A nearly identical DynaFit script was utilized to analyze the same data according to Method $\mathbf{B}$, where the the " $k_{\text {cat }}$ " rate constant labeled $k_{\text {d.I }}$ is treated as an optimized model parameter. The only difference between the two methods is the following line in the input script:

\section{Method A}

[constants]

...

$\mathrm{kd} . \mathrm{P}=0.036$

...

\section{Method B}

[constants]

...

$\mathrm{kd} . \mathrm{P}=0.036 ?$ 
Note that appending a question mark to the numerical value of any item listed in the DynaFit input script will transform it from a fixed constant to an adjustable or tunable model parameter.

\section{B. Systems of ODEs automatically derived by DynaFit}

This appendix contains systems of first-order ordinary differential equations (ODEs) automatically derived by the software package DynaFit [4] from symbolic input files listed in Appendix A.

\section{B.1. Substrate kinetics of SAM}

\section{B.1.1. Model MM}

The following system first-order ordinary differential equations describes the time course of an enzyme reaction following the simple Michaelis-Menten mechanism.

See section 1.2.1 for a symbolic representation.

$$
\begin{aligned}
\frac{\mathrm{d}[\mathrm{E}]}{\mathrm{d} t} & =-k_{\mathrm{a} . \mathrm{S}}[\mathrm{E}][\mathrm{S}]+k_{\mathrm{d} . \mathrm{S}}[\mathrm{E} . \mathrm{S}]+k_{\mathrm{d} . \mathrm{P}}[\mathrm{E} . \mathrm{S}] \\
\frac{\mathrm{d}[\mathrm{S}]}{\mathrm{d} t} & =-k_{\mathrm{a} . \mathrm{S}}[\mathrm{E}][\mathrm{S}]+k_{\mathrm{d} . \mathrm{S}}[\mathrm{E} . \mathrm{S}] \\
\frac{\mathrm{d}[\mathrm{E} . \mathrm{S}]}{\mathrm{d} t} & =+k_{\mathrm{a} . \mathrm{S}}[\mathrm{E}][\mathrm{S}]-k_{\mathrm{d} . \mathrm{S}}[\mathrm{E} . \mathrm{S}]-k_{\mathrm{d} . \mathrm{P}}[\mathrm{E} . \mathrm{S}] \\
\frac{\mathrm{d}[\mathrm{P}]}{\mathrm{d} t} & =+k_{\mathrm{d} . \mathrm{P}}[\mathrm{E} . \mathrm{S}]
\end{aligned}
$$

\section{B.1.2. Model $\mathbf{M M + p i}$}

The following system first-order ordinary differential equations describes the time course of an enzyme reaction following the Michaelis-Menten mechanism accompanied by product inhibition.

See section 1.2.2 for a symbolic representation.

$$
\begin{aligned}
\frac{\mathrm{d}[\mathrm{E}]}{\mathrm{d} t} & =-k_{\mathrm{a} . \mathrm{S}}[\mathrm{E}][\mathrm{S}]+k_{\mathrm{d} . \mathrm{S}}[\mathrm{E} . \mathrm{S}]+k_{\mathrm{d} . \mathrm{P}}[\mathrm{E} . \mathrm{S}]-k_{\mathrm{a} . \mathrm{Pi}}[\mathrm{E}][\mathrm{P}]+k_{\mathrm{d} . \mathrm{Pi}}[\mathrm{E} . \mathrm{P}] \\
\frac{\mathrm{d}[\mathrm{S}]}{\mathrm{d} t} & =-k_{\mathrm{a} . \mathrm{S}}[\mathrm{E}][\mathrm{S}]+k_{\mathrm{d} . \mathrm{S}}[\mathrm{E} . \mathrm{S}] \\
\frac{\mathrm{d}[\mathrm{E} . \mathrm{S}]}{\mathrm{d} t} & =+k_{\mathrm{a} . \mathrm{S}}[\mathrm{E}][\mathrm{S}]-k_{\mathrm{d} . \mathrm{S}}[\mathrm{E} . \mathrm{S}]-k_{\mathrm{d} . \mathrm{P}}[\mathrm{E} . \mathrm{S}] \\
\frac{\mathrm{d}[\mathrm{P}]}{\mathrm{d} t} & =+k_{\mathrm{d} . \mathrm{P}}[\mathrm{E} . \mathrm{S}]-k_{\mathrm{a} . \mathrm{Pi}}[\mathrm{E}][\mathrm{P}]+k_{\mathrm{d} . \mathrm{Pi}}[\mathrm{E} . \mathrm{P}] \\
\frac{\mathrm{d}[\mathrm{E} . \mathrm{P}]}{\mathrm{d} t} & =+k_{\mathrm{a} . \mathrm{Pi}}[\mathrm{E}][\mathrm{P}]-k_{\mathrm{d} . \mathrm{Pi}}[\mathrm{E} . \mathrm{P}]
\end{aligned}
$$




\section{B.1.3. Model MM+ed}

The following system first-order ordinary differential equations describes the time course of an enzyme reaction following the Michaelis-Menten mechanism accompanied by enzyme deactivation via fist-order decay of the Michaelis complex ES.

See section 1.2.3 for a symbolic representation.

$$
\begin{aligned}
\frac{\mathrm{d}[\mathrm{E}]}{\mathrm{d} t} & =-k_{\mathrm{a} . \mathrm{S}}[\mathrm{E}][\mathrm{S}]+k_{\mathrm{d} . \mathrm{S}}[\mathrm{E} . \mathrm{S}]+k_{\mathrm{d} . \mathrm{P}}[\mathrm{E} . \mathrm{S}] \\
\frac{\mathrm{d}[\mathrm{S}]}{\mathrm{d} t} & =-k_{\mathrm{a} . \mathrm{S}}[\mathrm{E}][\mathrm{S}]+k_{\mathrm{d} . \mathrm{S}}[\mathrm{E} . \mathrm{S}] \\
\frac{\mathrm{d}[\mathrm{E} . \mathrm{S}]}{\mathrm{d} t} & =+k_{\mathrm{a} . \mathrm{S}}[\mathrm{E}][\mathrm{S}]-k_{\mathrm{d} . \mathrm{S}}[\mathrm{E} . \mathrm{S}]-k_{\mathrm{d} . \mathrm{P}}[\mathrm{E} . \mathrm{S}]-k_{. \mathrm{ES}}[\mathrm{E} . \mathrm{S}] \\
\frac{\mathrm{d}[\mathrm{P}]}{\mathrm{d} t} & =+k_{\mathrm{d} . \mathrm{P}}[\mathrm{E} . \mathrm{S}] \\
\frac{\mathrm{d}[\mathrm{F}]}{\mathrm{d} t} & =+k_{. \mathrm{ES}}[\mathrm{E} . \mathrm{S}]
\end{aligned}
$$

\section{B.1.4. Model MM+pi+ed}

The following system first-order ordinary differential equations describes the time course of an enzyme reaction following the Michaelis-Menten mechanism accompanied by (a) product inhibition and (b) enzyme deactivation via fist-order decay of the Michaelis complex ES.

See section 1.2.4 for a symbolic representation.

$$
\begin{aligned}
\frac{\mathrm{d}[\mathrm{E}]}{\mathrm{d} t} & =-k_{\mathrm{a} . \mathrm{S}}[\mathrm{E}][\mathrm{S}]+k_{\mathrm{d} . \mathrm{S}}[\mathrm{E} . \mathrm{S}]+k_{\mathrm{d} . \mathrm{P}}[\mathrm{E} . \mathrm{S}]-k_{\mathrm{a} . \mathrm{Pi}}[\mathrm{E}][\mathrm{P}]+k_{\mathrm{d} . \mathrm{Pi}}[\mathrm{E} . \mathrm{P}] \\
\frac{\mathrm{d}[\mathrm{S}]}{\mathrm{d} t} & =-k_{\mathrm{a} . \mathrm{S}}[\mathrm{E}][\mathrm{S}]+k_{\mathrm{d} . \mathrm{S}}[\mathrm{E} . \mathrm{S}] \\
\frac{\mathrm{d}[\mathrm{E} . \mathrm{S}]}{\mathrm{d} t} & =+k_{\mathrm{a} . \mathrm{S}}[\mathrm{E}][\mathrm{S}]-k_{\mathrm{d} . \mathrm{S}}[\mathrm{E} . \mathrm{S}]-k_{\mathrm{d} . \mathrm{P}}[\mathrm{E} . \mathrm{S}]-k_{\mathrm{ES}}[\mathrm{E} . \mathrm{S}] \\
\frac{\mathrm{d}[\mathrm{P}]}{\mathrm{d} t} & =+k_{\mathrm{d} . \mathrm{P}}[\mathrm{E} . \mathrm{S}]-k_{\mathrm{a} . \mathrm{Pi}}[\mathrm{E}][\mathrm{P}]+k_{\mathrm{d} . \mathrm{Pi}}[\mathrm{E} . \mathrm{P}] \\
\frac{\mathrm{d}[\mathrm{E} . \mathrm{P}]}{\mathrm{d} t} & =+k_{\mathrm{a} . \mathrm{Pi}}[\mathrm{E}][\mathrm{P}]-k_{\mathrm{d} . \mathrm{Pi}}[\mathrm{E} . \mathrm{P}] \\
\frac{\mathrm{d}[\mathrm{F}]}{\mathrm{d} t} & =+k_{\text {.ES }}[\mathrm{E} . \mathrm{S}]
\end{aligned}
$$

\section{B.2. Inhibition kinetics}

\section{B.2.1. Models R1S and R1F}

The following system first-order ordinary differential equations describes the time course of an enzyme reaction following the Michaelis-Menten mechanism accompanied by (a) product inhibition and (b) enzyme deactivation via fist-order decay of the Michaelis complex ES. 
In addition, the inhibitor I binds to the enzyme $\mathrm{E}$ in a competitive manner (i.e., with mutual exclusion) with respect to the substrate S. Inhibitor binding is assumed to be reversible.

See section 4.2.2 for a symbolic representation of the mechanism.

$$
\begin{aligned}
\frac{\mathrm{d}[\mathrm{E}]}{\mathrm{d} t}= & -k_{\mathrm{a} . \mathrm{S}}[\mathrm{E}][\mathrm{S}]+k_{\mathrm{d} . \mathrm{S}}[\mathrm{E} . \mathrm{S}]+k_{\mathrm{d} . \mathrm{P}}[\mathrm{E} . \mathrm{S}]-k_{\mathrm{a} . \mathrm{Pi}}[\mathrm{E}][\mathrm{P}]+k_{\mathrm{d} . \mathrm{Pi}}[\mathrm{E} . \mathrm{P}] \\
& -k_{\mathrm{a} . \mathrm{I}}[\mathrm{E}][\mathrm{I}]+k_{\mathrm{d} . \mathrm{I}}[\mathrm{E} . \mathrm{I}] \\
\frac{\mathrm{d}[\mathrm{S}]}{\mathrm{d} t}= & -k_{\mathrm{a} . \mathrm{S}}[\mathrm{E}][\mathrm{S}]+k_{\mathrm{d} . \mathrm{S}}[\mathrm{E} . \mathrm{S}] \\
\frac{\mathrm{d}[\mathrm{E} . \mathrm{S}]}{\mathrm{d} t}= & +k_{\mathrm{a} . \mathrm{S}}[\mathrm{E}][\mathrm{S}]-k_{\mathrm{d} . \mathrm{S}}[\mathrm{E} . \mathrm{S}]-k_{\mathrm{d} . \mathrm{P}}[\mathrm{E} . \mathrm{S}]-k_{\mathrm{eS}}[\mathrm{E} . \mathrm{S}] \\
\frac{\mathrm{d}[\mathrm{P}]}{\mathrm{d} t}= & +k_{\mathrm{d} . \mathrm{P}}[\mathrm{E} . \mathrm{S}]-k_{\mathrm{a} . \mathrm{Pi}}[\mathrm{E}][\mathrm{P}]+k_{\mathrm{d} . \mathrm{P} i}[\mathrm{E} . \mathrm{P}] \\
\frac{\mathrm{d}[\mathrm{E} . \mathrm{P}]}{\mathrm{d} t}= & +k_{\mathrm{a} . \mathrm{Pi}}[\mathrm{E}][\mathrm{P}]-k_{\mathrm{d} . \mathrm{Pi}}[\mathrm{E} . \mathrm{P}] \\
\frac{\mathrm{d}[\mathrm{F}]}{\mathrm{d} t}= & +k_{. \mathrm{ES}}[\mathrm{E} . \mathrm{S}] \\
\frac{\mathrm{d}[\mathrm{I}]}{\mathrm{d} t}= & -k_{\mathrm{a} . \mathrm{I}}[\mathrm{E}][\mathrm{I}]+k_{\mathrm{d} . \mathrm{I}}[\mathrm{E} . \mathrm{I}] \\
\frac{\mathrm{d}[\mathrm{E} . \mathrm{I}]}{\mathrm{d} t}= & +k_{\mathrm{a} . \mathrm{I}}[\mathrm{E}][\mathrm{I}]-k_{\mathrm{d} . \mathrm{I}}[\mathrm{E} . \mathrm{I}]
\end{aligned}
$$

\section{B.2.2. Model C1}

The following system first-order ordinary differential equations describes the time course of an enzyme reaction following the Michaelis-Menten mechanism accompanied by (a) product inhibition and (b) enzyme deactivation via fist-order decay of the Michaelis complex ES.

In addition, the inhibitor I binds to the enzyme $\mathrm{E}$ in a competitive manner (i.e., with mutual exclusion) with respect to the substrate S. Inhibitor binding is assumed to be nominally irreversible.

In the physical as opposed to purely formal and mathematical domain, "irreversible" binding means that either the inhibitor is binding covalently or that the inhibitor is binding reversibly but, at the same time, the dissociation rate constant $k_{\mathrm{d} . \mathrm{I}}$ is so extremely low that the lower limit of its confidence interval, at the given confidence level, cannot be reliably determined from the given set of experimental data.

See section 4.2.4 for a symbolic representation of the mechanism. 


$$
\begin{aligned}
& \frac{\mathrm{d}[\mathrm{E}]}{\mathrm{d} t}=-k_{\mathrm{a} . \mathrm{S}}[\mathrm{E}][\mathrm{S}]+k_{\mathrm{d} . \mathrm{S}}[\mathrm{E} . \mathrm{S}]+k_{\mathrm{d} . \mathrm{P}}[\mathrm{E} . \mathrm{S}]-k_{\mathrm{a} . \mathrm{Pi}}[\mathrm{E}][\mathrm{P}]+k_{\mathrm{d} . \mathrm{Pi}}[\mathrm{E} . \mathrm{P}] \\
& -k_{\mathrm{a} . \mathrm{I}}[\mathrm{E}][\mathrm{I}] \\
& \frac{\mathrm{d}[\mathrm{S}]}{\mathrm{d} t}=-k_{\mathrm{a} . \mathrm{S}}[\mathrm{E}][\mathrm{S}]+k_{\mathrm{d} . \mathrm{S}}[\mathrm{E} . \mathrm{S}] \\
& \frac{\mathrm{d}[\mathrm{E} . \mathrm{S}]}{\mathrm{d} t}=+k_{\mathrm{a} . \mathrm{S}}[\mathrm{E}][\mathrm{S}]-k_{\mathrm{d} . \mathrm{S}}[\mathrm{E} . \mathrm{S}]-k_{\mathrm{d} . \mathrm{P}}[\mathrm{E} . \mathrm{S}]-k_{\text {.ES }}[\mathrm{E} . \mathrm{S}] \\
& \frac{\mathrm{d}[\mathrm{P}]}{\mathrm{d} t}=+k_{\mathrm{d} . \mathrm{P}}[\mathrm{E} . \mathrm{S}]-k_{\mathrm{a} . \mathrm{Pi}}[\mathrm{E}][\mathrm{P}]+k_{\mathrm{d} . \mathrm{Pi}}[\mathrm{E} . \mathrm{P}] \\
& \frac{\mathrm{d}[\mathrm{E} . \mathrm{P}]}{\mathrm{d} t}=+k_{\mathrm{a} . \mathrm{Pi}}[\mathrm{E}][\mathrm{P}]-k_{\mathrm{d} . \mathrm{Pi}}[\mathrm{E} . \mathrm{P}] \\
& \frac{\mathrm{d}[\mathrm{F}]}{\mathrm{d} t}=+k_{\text {ES }}[\mathrm{E} . \mathrm{S}] \\
& \frac{\mathrm{d}[\mathrm{I}]}{\mathrm{d} t}=-k_{\mathrm{a} . \mathrm{I}}[\mathrm{E}][\mathrm{I}] \\
& \frac{\mathrm{d}[\mathrm{E} \cdot \mathrm{I}]}{\mathrm{d} t}=+k_{\mathrm{a} . \mathrm{I}}[\mathrm{E}][\mathrm{I}]
\end{aligned}
$$

\section{Model-selection results}

This appendix contains model-selection results automatically derived by the software package DynaFit [4] from symbolic input files listed in Appendix A.

In the auto-generated tables below, the meaning of mathematical symbols that appear in the table headings is as follows:

Value best-fit value of the given model parameter

StdErr formal standard error of each parameter computed from diagonal elements of the covariance matrix

$\mathrm{CV} \%$ coefficient of variation

Low lower limit of the confidence interval

High upper limit of the confidence interval

$\mathrm{P}$ (Low) probability level reached at the lower bound of the confidence interval

$\mathrm{P}(\mathrm{High})$ probability level reached at the upper bound of the confidence interval

$n_{\mathrm{P}}$ number of adjustable model parameters

$S S Q_{\mathrm{r}} \quad$ relative sum of squared deviations

$\triangle A I C$ differential Akaike Information Criterion

$\triangle B I C \quad$ differential Bayesian Information Criterion

$w^{\text {(AIC) }} \quad$ Akaike weight

$w^{(\mathrm{BIC})} \quad$ Bayesian weight

Model failures - Legend 
:Delta ... Differential AIC/BIC criterion greater than 5

:Weight ... Akaike / Bayesian weight lower than 0.01

:Squares ... Relative sum of squared deviations greater than 1.05

For further details regarding the mathematical and statistical principles of model discrimination implemented in the DynaFit software pacakge,, see refs. [4, 11-13].

C.1. Substrate kinetics of SAM

\section{C.1.1. Replicate RI}

Intermediate results

Kinetic constants

\begin{tabular}{llrrrrrrr}
\hline Model & Parameter & Value & StdErr & CV \% & Low & High & P(Low) \% & P(High) \% \\
\hline MM & kd.S & 21.3 & 2.1 & 19.1 & 18 & 25.3 & 96.3 & 96.3 \\
& kd.P & 0.0348 & 0.0028 & 15.5 & 0.029 & 0.04 & 96.3 & 96.3 \\
MM+Pi & kd.S & 0.0588 & 0.0045 & 14.3 & 0.05 & 0.0691 & 96.3 & 96.3 \\
& kd.P & 0.0108 & 0.0017 & 29.5 & 0.00926 & 0.0125 & 96.3 & 96.3 \\
& kd.Pi & 0.00366 & 0.00065 & 33.0 & 0.00304 & 0.00448 & 96.3 & 96.3 \\
MM+Ed & kd.S & 44.4 & 2.8 & 11.9 & 39.8 & 49.8 & 96.3 & 96.3 \\
& kd.P & 0.0523 & 0.0026 & 9.3 & 0.0468 & 0.0575 & 96.3 & 96.3 \\
& k.ES & 0.00128 & $5.1 \mathrm{e}-005$ & 7.4 & 0.00119 & 0.00138 & 96.3 & 96.3 \\
MM+Pi+Ed & kd.S & 8 & 1 & 22.4 & 6.07 & 10 & 96.2 & 96.2 \\
& kd.P & 0.0301 & 0.00086 & 5.1 & 0.0283 & 0.0319 & 96.2 & 96.2 \\
& kd.Pi & 0.984 & 0.15 & 27.8 & 0.701 & 1.32 & 96.2 & 96.2 \\
& k.ES & 0.000678 & $2.3 \mathrm{e}-005$ & 6.2 & 0.00063 & 0.000727 & 96.2 & 96.2 \\
\hline
\end{tabular}

Information-Theoretic Criteria - Full Set

\begin{tabular}{lrrrrrrl}
\hline model & $n_{\mathrm{P}}$ & $S S Q_{\mathrm{r}}$ & $\Delta A I C$ & $\Delta B I C$ & $w^{(\mathrm{AIC})}$ & $w^{(\mathrm{BIC})}$ & parameters \\
\hline $\mathrm{MM}+\mathrm{Pi}+\mathrm{Ed}$ & 15 & 1.000 & 0.0 & 0.0 & 1.000 & 1.000 & OK \\
$\mathrm{MM}+\mathrm{Pi}$ & 14 & 2.796 & 460.5 & 456.3 & 0.000 & 0.000 & OK \\
MM+Ed & 14 & 6.099 & 811.5 & 807.4 & 0.000 & 0.000 & OK \\
MM & 13 & 24.770 & 1440.0 & 1431.8 & 0.000 & 0.000 & OK \\
\hline
\end{tabular}

Final results

Information-Theoretic Criteria - Reduced Set

\begin{tabular}{lrrrrrrl}
\hline model & $n_{\mathrm{P}}$ & $S S Q_{\mathrm{r}}$ & $\Delta A I C$ & $\Delta B I C$ & $w^{(\mathrm{AIC})}$ & $w^{(\mathrm{BIC})}$ & status \\
\hline $\mathrm{MM}+\mathrm{Pi}+\mathrm{Ed}$ & 15 & 1.000 & 0.0 & 0.0 & 1.000 & 1.000 & OK \\
$\mathrm{MM}+\mathrm{Pi}$ & 14 & 2.796 & 460.5 & 456.3 & 0.000 & 0.000 & FAIL:Squares \\
$\mathrm{MM}+\mathrm{Ed}$ & 14 & 6.099 & 811.5 & 807.4 & 0.000 & 0.000 & FAIL:Squares \\
$\mathrm{MM}$ & 13 & 24.770 & 1440.0 & 1431.8 & 0.000 & 0.000 & FAIL:Squares \\
\hline
\end{tabular}


Model Discrimination Analysis - Conclusions

A single candidate model passes all acceptance criteria:

\begin{tabular}{rrrrrr}
\hline task no. & model ID & $n_{P}$ & $\mathrm{SSQ}_{r}$ & $\Delta \mathrm{AIC}$ & $\Delta \mathrm{BIC}$ \\
\hline 4 & $\mathrm{MM}+\mathrm{Pi}+\mathrm{Ed}$ & 15 & 1 & 0 & 0 \\
\hline
\end{tabular}

Acceptable parameters

\begin{tabular}{llrrr}
\hline Model & Parameter & Value & Low & High \\
\hline MM+Pi+Ed & kd.S & 8 & 6.07 & 10 \\
& $\mathrm{kd.P}$ & 0.0301 & 0.0283 & 0.0319 \\
& $\mathrm{kd.Pi}$ & 0.984 & 0.701 & 1.32 \\
& $\mathrm{k} . \mathrm{ES}$ & 0.000678 & 0.00063 & 0.000727 \\
\hline
\end{tabular}

C.1.2. Replicate $R 2$

Intermediate results

Kinetic constants

\begin{tabular}{llrrrrrrr}
\hline Model & Parameter & Value & StdErr & CV \% & Low & High & P(Low) \% & P(High) \% \\
\hline MM & kd.S & 22.9 & 1.9 & 16.1 & 19.7 & 26.5 & 96.3 & 96.3 \\
& kd.P & 0.0398 & 0.0024 & 11.6 & 0.0348 & 0.0444 & 96.3 & 96.3 \\
MM+Pi & kd.S & 0.0636 & 0.0069 & 20.1 & 0.0512 & 0.0819 & 96.3 & 96.3 \\
& kd.P & 0.0192 & 0.001 & 9.9 & 0.0169 & 0.0214 & 96.3 & 96.3 \\
& kd.Pi & 0.00789 & 0.00091 & 21.6 & 0.00618 & 0.0104 & 96.3 & 96.3 \\
MM+Ed & kd.S & 44.4 & 2.3 & 9.7 & 40.5 & 48.9 & 96.3 & 96.3 \\
& kd.P & 0.0562 & 0.0021 & 6.9 & 0.0518 & 0.0604 & 96.3 & 96.3 \\
& k.ES & 0.0011 & $3.9 \mathrm{e}-005$ & 6.7 & 0.00103 & 0.00118 & 96.3 & 96.3 \\
$\mathrm{MM}+\mathrm{Pi}+\mathrm{Ed}$ & $\mathrm{kd} . \mathrm{S}$ & 12.8 & 0.94 & 13.2 & 10.9 & 14.8 & 96.2 & 96.2 \\
& $\mathrm{kd.P}$ & 0.0376 & 0.00079 & 3.8 & 0.036 & 0.0393 & 96.2 & 96.2 \\
& $\mathrm{k} . P i$ & 2.69 & 0.28 & 18.5 & 2.15 & 3.3 & 96.2 & 96.2 \\
& $\mathrm{k} . \mathrm{ES}$ & 0.000542 & $2.1 \mathrm{e}-005$ & 7.0 & 0.000499 & 0.000586 & 96.2 & 96.2 \\
\hline
\end{tabular}

Information-Theoretic Criteria - Full Set

\begin{tabular}{lrrrrrrl}
\hline model & $n_{\mathrm{P}}$ & $S S Q_{\mathrm{r}}$ & $\Delta A I C$ & $\Delta B I C$ & $w^{(\mathrm{AIC})}$ & $w^{(\mathrm{BIC})}$ & parameters \\
\hline $\mathrm{MM}+\mathrm{Pi}+\mathrm{Ed}$ & 15 & 1.000 & 0.0 & 0.0 & 1.000 & 1.000 & OK \\
$\mathrm{MM}+\mathrm{Pi}$ & 14 & 2.316 & 375.8 & 371.7 & 0.000 & 0.000 & OK \\
$\mathrm{MM}+\mathrm{Ed}$ & 14 & 5.265 & 745.3 & 741.2 & 0.000 & 0.000 & OK \\
$\mathrm{MM}$ & 13 & 22.847 & 1403.7 & 1395.5 & 0.000 & 0.000 & OK \\
\hline
\end{tabular}

Final results

Information-Theoretic Criteria - Reduced Set

\begin{tabular}{lrrrrrrl}
\hline model & $n_{\mathrm{P}}$ & $S S Q_{\mathrm{r}}$ & $\Delta A I C$ & $\Delta B I C$ & $w^{(\mathrm{AIC})}$ & $w^{(\mathrm{BIC})}$ & status \\
\hline $\mathrm{MM}+\mathrm{Pi}+\mathrm{Ed}$ & 15 & 1.000 & 0.0 & 0.0 & 1.000 & 1.000 & OK \\
MM+Pi & 14 & 2.316 & 375.8 & 371.7 & 0.000 & 0.000 & FAIL:Squares \\
MM+Ed & 14 & 5.265 & 745.3 & 741.2 & 0.000 & 0.000 & FAIL:Squares \\
MM & 13 & 22.847 & 1403.7 & 1395.5 & 0.000 & 0.000 & FAIL:Squares \\
\hline
\end{tabular}


Model Discrimination Analysis - Conclusions

A single candidate model passes all acceptance criteria:

\begin{tabular}{rrrrrr}
\hline task no. & model ID & $n_{P}$ & $\mathrm{SSQ}_{r}$ & $\Delta \mathrm{AIC}$ & $\Delta \mathrm{BIC}$ \\
\hline 4 & $\mathrm{MM}+\mathrm{Pi}+\mathrm{Ed}$ & 15 & 1 & 0 & 0 \\
\hline
\end{tabular}

Acceptable parameters

\begin{tabular}{llrrr}
\hline Model & Parameter & Value & Low & High \\
\hline MM+Pi+Ed & kd.S & 12.8 & 10.9 & 14.8 \\
& kd.P & 0.0376 & 0.036 & 0.0393 \\
& kd.Pi & 2.69 & 2.15 & 3.3 \\
& k.ES & 0.000542 & 0.000499 & 0.000586 \\
\hline
\end{tabular}

C.1.3. Replicate R3

Intermediate results

Kinetic constants

\begin{tabular}{llrrrrrrr}
\hline Model & Parameter & Value & StdErr & CV \% & Low & High & P(Low) \% & P(High) \% \\
\hline MM & kd.S & 24.8 & 2.1 & 16.3 & 21.5 & 28.7 & 96.3 & 96.3 \\
& kd.P & 0.043 & 0.0025 & 11.4 & 0.0376 & 0.0479 & 96.3 & 96.3 \\
MM+Pi & kd.S & 0.0991 & 0.016 & 30.9 & 0.0681 & 0.19 & 96.3 & 96.3 \\
& kd.P & 0.0239 & 0.001 & 8.1 & 0.0213 & 0.0267 & 96.3 & 96.3 \\
& kd.Pi & 0.013 & 0.002 & 28.9 & 0.00896 & 0.0241 & 96.3 & 96.3 \\
MM+Ed & kd.S & 48.9 & 2.6 & 9.8 & 44.5 & 53.7 & 96.3 & 96.3 \\
& kd.P & 0.0611 & 0.0022 & 6.8 & 0.0565 & 0.0656 & 96.3 & 96.3 \\
& k.ES & 0.00113 & $4.1 \mathrm{e}-005$ & 6.8 & 0.00105 & 0.00121 & 96.3 & 96.3 \\
$\mathrm{MM}+\mathrm{Pi}+\mathrm{Ed}$ & $\mathrm{kd} . \mathrm{S}$ & 15.8 & 1 & 11.5 & 13.8 & 17.9 & 96.2 & 96.2 \\
& $\mathrm{kd.P}$ & 0.0411 & 0.00084 & 3.7 & 0.0393 & 0.0428 & 96.2 & 96.2 \\
& $\mathrm{k} . P i$ & 3.29 & 0.31 & 16.7 & 2.7 & 3.96 & 96.2 & 96.2 \\
& $\mathrm{k} . \mathrm{ES}$ & 0.000543 & $2.2 \mathrm{e}-005$ & 7.2 & 0.000498 & 0.000588 & 96.2 & 96.2 \\
\hline
\end{tabular}

Information-Theoretic Criteria - Full Set

\begin{tabular}{lrrrrrrl}
\hline model & $n_{\mathrm{P}}$ & $S S Q_{\mathrm{r}}$ & $\Delta A I C$ & $\Delta B I C$ & $w^{(\mathrm{AIC})}$ & $w^{(\mathrm{BIC})}$ & parameters \\
\hline $\mathrm{MM}+\mathrm{Pi}+\mathrm{Ed}$ & 15 & 1.000 & 0.0 & 0.0 & 1.000 & 1.000 & OK \\
$\mathrm{MM}+\mathrm{Pi}$ & 14 & 2.415 & 394.6 & 390.5 & 0.000 & 0.000 & OK \\
$\mathrm{MM}+\mathrm{Ed}$ & 14 & 5.415 & 758.0 & 753.9 & 0.000 & 0.000 & OK \\
$\mathrm{MM}$ & 13 & 24.028 & 1426.4 & 1418.1 & 0.000 & 0.000 & OK \\
\hline
\end{tabular}

Final results

Information-Theoretic Criteria - Reduced Set

\begin{tabular}{lrrrrrrl}
\hline model & $n_{\mathrm{P}}$ & $S S Q_{\mathrm{r}}$ & $\Delta A I C$ & $\Delta B I C$ & $w^{(\mathrm{AIC})}$ & $w^{(\mathrm{BIC})}$ & status \\
\hline $\mathrm{MM}+\mathrm{Pi}+\mathrm{Ed}$ & 15 & 1.000 & 0.0 & 0.0 & 1.000 & 1.000 & OK \\
$\mathrm{MM}+\mathrm{Pi}$ & 14 & 2.415 & 394.6 & 390.5 & 0.000 & 0.000 & FAIL:Squares \\
$\mathrm{MM}+\mathrm{Ed}$ & 14 & 5.415 & 758.0 & 753.9 & 0.000 & 0.000 & FAIL:Squares \\
$\mathrm{MM}$ & 13 & 24.028 & 1426.4 & 1418.1 & 0.000 & 0.000 & FAIL:Squares \\
\hline
\end{tabular}


Model Discrimination Analysis - Conclusions

A single candidate model passes all acceptance criteria:

\begin{tabular}{rrrrrr}
\hline task no. & model ID & $n_{P}$ & $\mathrm{SSQ}_{r}$ & $\Delta \mathrm{AIC}$ & $\Delta \mathrm{BIC}$ \\
\hline 4 & $\mathrm{MM}+\mathrm{Pi}+\mathrm{Ed}$ & 15 & 1 & 0 & 0 \\
\hline
\end{tabular}

Acceptable parameters

\begin{tabular}{llrrr}
\hline Model & Parameter & Value & Low & High \\
\hline MM+Pi+Ed & kd.S & 15.8 & 13.8 & 17.9 \\
& kd.P & 0.0411 & 0.0393 & 0.0428 \\
& kd.Pi & 3.29 & 2.7 & 3.96 \\
& k.ES & 0.000543 & 0.000498 & 0.000588 \\
\hline
\end{tabular}

\section{C.2. Inhibitor kinetics of compound $\mathbf{1 8}$}

Intermediate results

Kinetic constants

\begin{tabular}{llrrrrrrr}
\hline Model & Parameter & Value & StdErr & CV \% & Low & High & P(Low) \% & P(High) \% \\
\hline R1S & ka.I & $5.35 \mathrm{e}+006$ & $3.1 \mathrm{e}+008$ & 5906.1 & 0.0837 & $1.68 \mathrm{e}+008$ & 74.2 & 0 \\
& kd.I & $1.8 \mathrm{e}+007$ & $1 \mathrm{e}+009$ & 5908.3 & 0.28 & $2 \mathrm{e}+006$ & 74.2 & 0 \\
R1F & kd.I & 33.5 & 2 & 6.4 & 31.4 & 35.8 & 74.4 & 74.4 \\
C1 & ka.I & 0.00116 & $8.2 \mathrm{e}-005$ & 7.4 & 0.00107 & 0.00126 & 74.4 & 74.4 \\
\hline
\end{tabular}

Information-Theoretic Criteria - Full Set

\begin{tabular}{lrrrrrrl}
\hline model & $n_{\mathrm{P}}$ & $S S Q_{\mathrm{r}}$ & $\Delta A I C$ & $\Delta B I C$ & $w^{(\mathrm{AIC})}$ & $w^{(\mathrm{BIC})}$ & parameters \\
\hline $\mathrm{R} 1 \mathrm{~F}$ & 13 & 1.000 & 0.0 & 0.0 & 0.776 & 0.938 & OK \\
$\mathrm{R} 1 \mathrm{~S}$ & 14 & 1.000 & 2.5 & 5.4 & 0.224 & 0.062 & FAIL:UB \\
$\mathrm{C} 1$ & 13 & 1.885 & 90.6 & 90.6 & 0.000 & 0.000 & OK \\
\hline
\end{tabular}

Parameter failures - Legend

:LB ... Missing lower bound for at least one parameter

:UB ... Missing upper bound

:UB/LB ... Upper-to-lower bound ratio too large

:CV ... Coefficient of variation too large

Final results

Information-Theoretic Criteria - Reduced Set

\begin{tabular}{lrrrrrrl}
\hline model & $n_{\mathrm{P}}$ & $S S Q_{\mathrm{r}}$ & $\Delta A I C$ & $\Delta B I C$ & $w^{(\mathrm{AIC})}$ & $w^{(\mathrm{BIC})}$ & status \\
\hline $\mathrm{R} 1 \mathrm{~F}$ & 13 & 1.000 & 0.0 & 0.0 & 1.000 & 1.000 & OK \\
$\mathrm{C} 1$ & 13 & 1.884 & 90.6 & 90.6 & 0.000 & 0.000 & FAIL:Squares \\
\hline
\end{tabular}


Model failures - Legend

:Delta ... Differential AIC/BIC criterion greater than 5

:Weight ... Akaike / Bayesian weight lower than 0.01

:Squares ... Relative sum of squared deviations greater than 1.05

Model Discrimination Analysis - Conclusions

A single candidate model passes all acceptance criteria:

\begin{tabular}{rrrrrr}
\hline task no. & model ID & $n_{P}$ & $\mathrm{SSQ}_{r}$ & $\Delta \mathrm{AIC}$ & $\Delta \mathrm{BIC}$ \\
\hline 2 & $\mathrm{R} 1 \mathrm{~F}$ & 13 & 1.00008 & 0 & 0 \\
\hline
\end{tabular}

Acceptable parameters

\begin{tabular}{llrrr}
\hline Model & Parameter & Value & Low & High \\
\hline R1F & kd.I & 33.5 & 31.4 & 35.8 \\
\hline
\end{tabular}

C.3. Inhibitor kinetics of compound $\mathbf{2 6}$

Intermediate results

Kinetic constants

\begin{tabular}{llrrrrrrr}
\hline Model & Parameter & Value & StdErr & CV \% & Low & High & P(Low) \% & P(High) \% \\
\hline R1S & ka.I & 0.365 & 0.0096 & 5.0 & 0.347 & 0.383 & 93.7 & 93.7 \\
& kd.I & 0.000134 & $3.8 \mathrm{e}-005$ & 53.0 & $6.39 \mathrm{e}-005$ & 0.000207 & 93.7 & 93.7 \\
R1F & kd.I & 0.0235 & 0.00096 & 8.0 & 0.0218 & 0.0254 & 93.8 & 93.8 \\
C1 & ka.I & 0.335 & 0.0038 & 2.2 & 0.328 & 0.342 & 93.8 & 93.8 \\
\hline
\end{tabular}

Information-Theoretic Criteria - Full Set

\begin{tabular}{lrrrrrrl}
\hline model & $n_{\mathrm{P}}$ & $S S Q_{\mathrm{r}}$ & $\Delta A I C$ & $\Delta B I C$ & $w^{(\mathrm{AIC})}$ & $w^{(\mathrm{BIC})}$ & parameters \\
\hline $\mathrm{R} 1 \mathrm{~S}$ & 11 & 1.000 & 0.0 & 0.0 & 0.996 & 0.973 & $\mathrm{OK}$ \\
$\mathrm{C} 1$ & 10 & 1.037 & 11.1 & 7.2 & 0.004 & 0.027 & $\mathrm{OK}$ \\
$\mathrm{R} 1 \mathrm{~F}$ & 10 & 7.475 & 722.0 & 718.1 & 0.000 & 0.000 & $\mathrm{OK}$ \\
\hline
\end{tabular}

Final results

Information-Theoretic Criteria - Reduced Set

\begin{tabular}{lrrrrrrl}
\hline model & $n_{\mathrm{P}}$ & $S S Q_{\mathrm{r}}$ & $\Delta A I C$ & $\Delta B I C$ & $w^{(\mathrm{AIC})}$ & $w^{(\mathrm{BIC})}$ & status \\
\hline $\mathrm{R} 1 \mathrm{~S}$ & 11 & 1.000 & 0.0 & 0.0 & 0.996 & 0.973 & OK \\
$\mathrm{C} 1$ & 10 & 1.037 & 11.1 & 7.2 & 0.004 & 0.027 & OK \\
$\mathrm{R} 1 \mathrm{~F}$ & 10 & 7.475 & 722.0 & 718.1 & 0.000 & 0.000 & FAIL:Squares \\
\hline
\end{tabular}

Model failures - Legend

:Delta ... Differential AIC/BIC criterion greater than 5

:Weight ... Akaike / Bayesian weight lower than 0.01

:Squares ... Relative sum of squared deviations greater than 1.05 
Model Discrimination Analysis - Conclusions

2 Different candidate models pass all acceptance criteria:

\begin{tabular}{rrrrrr}
\hline task no. & model ID & $n_{P}$ & $\mathrm{SSQ}_{r}$ & $\Delta \mathrm{AIC}$ & $\Delta \mathrm{BIC}$ \\
\hline 1 & $\mathrm{R} 1 \mathrm{~S}$ & 11 & 1 & 0 & 0 \\
3 & $\mathrm{C} 1$ & 10 & 1.03742 & 11.0862 & 7.20007 \\
\hline
\end{tabular}

Acceptable parameters

\begin{tabular}{llrrr}
\hline Model & Parameter & Value & Low & High \\
\hline R1S & ka.I & 0.365 & 0.347 & 0.383 \\
& kd.I & 0.000134 & $6.39 \mathrm{e}-005$ & 0.000207 \\
C1 & ka.I & 0.335 & 0.328 & 0.342 \\
\hline
\end{tabular}

C.4. Inhibitor kinetics of compound $\mathbf{3 1}$

C.4.1. Replicate 1

Intermediate results

Kinetic constants

\begin{tabular}{llrrrrrrr}
\hline Model & Parameter & Value & StdErr & CV \% & Low & High & P(Low) \% & P(High) \% \\
\hline R1S & ka.I & 4.96 & 3.4 & 68.8 & 2.75 & 16.4 & 66.3 & 66.3 \\
& kd.I & 0.0168 & 0.012 & 73.2 & 0.00871 & 0.0575 & 66.3 & 66.3 \\
R1F & kd.I & 0.0348 & 0.0033 & 9.6 & 0.0317 & 0.038 & 66.5 & 66.5 \\
C1 & ka.I & 0.818 & 0.063 & 7.9 & 0.755 & 0.887 & 66.5 & 66.5 \\
\hline
\end{tabular}

Information-Theoretic Criteria - Full Set

\begin{tabular}{lrrrrrrl}
\hline model & $n_{\mathrm{P}}$ & $S S Q_{\mathrm{r}}$ & $\Delta A I C$ & $\Delta B I C$ & $w^{(\mathrm{AIC})}$ & $w^{(\mathrm{BIC})}$ & parameters \\
\hline $\mathrm{R} 1 \mathrm{~F}$ & 10 & 1.005 & 0.0 & 0.0 & 0.735 & 0.912 & OK \\
$\mathrm{R} 1 \mathrm{~S}$ & 11 & 1.000 & 2.0 & 4.7 & 0.265 & 0.088 & $\mathrm{OK}$ \\
$\mathrm{C} 1$ & 10 & 1.355 & 31.1 & 31.1 & 0.000 & 0.000 & $\mathrm{OK}$ \\
\hline
\end{tabular}

Final results

Information-Theoretic Criteria - Reduced Set

\begin{tabular}{lrrrrrrl}
\hline model & $n_{\mathrm{P}}$ & $S S Q_{\mathrm{r}}$ & $\Delta A I C$ & $\Delta B I C$ & $w^{(\mathrm{AIC})}$ & $w^{(\mathrm{BIC})}$ & status \\
\hline $\mathrm{R} 1 \mathrm{~F}$ & 10 & 1.005 & 0.0 & 0.0 & 0.735 & 0.912 & OK \\
$\mathrm{R} 1 \mathrm{~S}$ & 11 & 1.000 & 2.0 & 4.7 & 0.265 & 0.088 & OK \\
$\mathrm{C} 1$ & 10 & 1.355 & 31.1 & 31.1 & 0.000 & 0.000 & FAIL:Squares \\
\hline
\end{tabular}

Model failures - Legend

:Delta ... Differential AIC/BIC criterion greater than 5

:Weight ... Akaike / Bayesian weight lower than 0.01

:Squares ... Relative sum of squared deviations greater than 1.05 
Model Discrimination Analysis - Conclusions

2 Different candidate models pass all acceptance criteria:

\begin{tabular}{rrrrrr}
\hline task no. & model ID & $n_{P}$ & $\mathrm{SSQ}_{r}$ & $\Delta \mathrm{AIC}$ & $\Delta \mathrm{BIC}$ \\
\hline 1 & $\mathrm{R} 1 \mathrm{~S}$ & 11 & 1 & 2.03538 & 4.67977 \\
2 & $\mathrm{R} 1 \mathrm{~F}$ & 10 & 1.00505 & 0 & 0 \\
\hline
\end{tabular}

Acceptable parameters

\begin{tabular}{llrrr}
\hline Model & Parameter & Value & Low & High \\
\hline R1S & ka.I & 4.96 & 2.75 & 16.4 \\
& kd.I & 0.0168 & 0.00871 & 0.0575 \\
R1F & kd.I & 0.0348 & 0.0317 & 0.038 \\
\hline
\end{tabular}

C.4.2. Replicate 2

Intermediate results

Kinetic constants

\begin{tabular}{llrrrrrrr}
\hline Model & Parameter & Value & StdErr & CV \% & Low & High & P(Low) \% & P(High) \% \\
\hline R1S & ka.I & 2.57 & 0.96 & 37.4 & 1.85 & 4 & 66.3 & 66.3 \\
& kd.I & 0.00968 & 0.0043 & 44.2 & 0.00644 & 0.0158 & 66.3 & 66.3 \\
R1F & kd.I & 0.0407 & 0.0034 & 8.7 & 0.0374 & 0.0443 & 66.5 & 66.5 \\
C1 & ka.I & 0.707 & 0.05 & 7.2 & 0.656 & 0.764 & 66.5 & 66.5 \\
\hline
\end{tabular}

Information-Theoretic Criteria - Full Set

\begin{tabular}{lrrrrrrl}
\hline model & $n_{\mathrm{P}}$ & $S S Q_{\mathrm{r}}$ & $\Delta A I C$ & $\Delta B I C$ & $w^{(\mathrm{AIC})}$ & $w^{(\mathrm{BIC})}$ & parameters \\
\hline $\mathrm{R} 1 \mathrm{~S}$ & 11 & 1.000 & 0.0 & 0.1 & 0.779 & 0.485 & $\mathrm{OK}$ \\
$\mathrm{R} 1 \mathrm{~F}$ & 10 & 1.050 & 2.5 & 0.0 & 0.221 & 0.515 & $\mathrm{OK}$ \\
$\mathrm{C} 1$ & 10 & 1.423 & 34.1 & 31.6 & 0.000 & 0.000 & $\mathrm{OK}$ \\
\hline
\end{tabular}

Final results

Information-Theoretic Criteria - Reduced Set

\begin{tabular}{lrrrrrrl}
\hline model & $n_{\mathrm{P}}$ & $S S Q_{\mathrm{r}}$ & $\Delta A I C$ & $\Delta B I C$ & $w^{(\mathrm{AIC})}$ & $w^{(\mathrm{BIC})}$ & status \\
\hline $\mathrm{R} 1 \mathrm{~S}$ & 11 & 1.000 & 0.0 & 0.1 & 0.779 & 0.485 & OK \\
$\mathrm{R} 1 \mathrm{~F}$ & 10 & 1.050 & 2.5 & 0.0 & 0.221 & 0.515 & FAIL:Squares \\
$\mathrm{C} 1$ & 10 & 1.423 & 34.1 & 31.6 & 0.000 & 0.000 & FAIL:Squares \\
\hline
\end{tabular}

Model failures - Legend

:Delta ... Differential AIC/BIC criterion greater than 5

:Weight ... Akaike / Bayesian weight lower than 0.01

:Squares ... Relative sum of squared deviations greater than 1.05 
Model Discrimination Analysis - Conclusions

A single candidate model passes all acceptance criteria:

\begin{tabular}{rrrrrr}
\hline task no. & model ID & $n_{P}$ & $\mathrm{SSQ}_{r}$ & $\Delta \mathrm{AIC}$ & $\Delta \mathrm{BIC}$ \\
\hline 1 & $\mathrm{R} 1 \mathrm{~S}$ & 11 & 1 & 0 & 0.123159 \\
\hline
\end{tabular}

Acceptable parameters

\begin{tabular}{llrrr}
\hline Model & Parameter & Value & Low & High \\
\hline R1S & ka.I & 2.57 & 1.85 & 4 \\
& kd.I & 0.00968 & 0.00644 & 0.0158 \\
\hline
\end{tabular}

C.4.3. Replicate 3

Intermediate results

Kinetic constants

\begin{tabular}{llrrrrrrr}
\hline Model & Parameter & Value & StdErr & CV \% & Low & High & P(Low) \% & P(High) \% \\
\hline R1S & ka.I & 3.41 & 1.2 & 35.7 & 2.5 & 5.24 & 66.3 & 66.3 \\
& kd.I & 0.00845 & 0.0036 & 42.6 & 0.0057 & 0.0137 & 66.3 & 66.3 \\
R1F & kd.I & 0.0268 & 0.0023 & 8.9 & 0.0247 & 0.0291 & 66.5 & 66.5 \\
C1 & ka.I & 0.994 & 0.07 & 7.2 & 0.925 & 1.07 & 66.5 & 66.5 \\
\hline
\end{tabular}

Information-Theoretic Criteria - Full Set

\begin{tabular}{lrrrrrrl}
\hline model & $n_{\mathrm{P}}$ & $S S Q_{\mathrm{r}}$ & $\Delta A I C$ & $\Delta B I C$ & $w^{(\mathrm{AIC})}$ & $w^{(\mathrm{BIC})}$ & parameters \\
\hline $\mathrm{R} 1 \mathrm{~S}$ & 11 & 1.000 & 0.0 & 1.4 & 0.653 & 0.334 & $\mathrm{OK}$ \\
$\mathrm{R} 1 \mathrm{~F}$ & 10 & 1.037 & 1.3 & 0.0 & 0.347 & 0.666 & $\mathrm{OK}$ \\
$\mathrm{C} 1$ & 10 & 1.414 & 33.5 & 32.2 & 0.000 & 0.000 & $\mathrm{OK}$ \\
\hline
\end{tabular}

Final results

Information-Theoretic Criteria - Reduced Set

\begin{tabular}{lrrrrrrl}
\hline model & $n_{\mathrm{P}}$ & $S S Q_{\mathrm{r}}$ & $\Delta A I C$ & $\Delta B I C$ & $w^{(\mathrm{AIC})}$ & $w^{(\mathrm{BIC})}$ & status \\
\hline $\mathrm{R} 1 \mathrm{~S}$ & 11 & 1.000 & 0.0 & 1.4 & 0.653 & 0.334 & OK \\
$\mathrm{R} 1 \mathrm{~F}$ & 10 & 1.037 & 1.3 & 0.0 & 0.347 & 0.666 & OK \\
$\mathrm{C} 1$ & 10 & 1.414 & 33.5 & 32.2 & 0.000 & 0.000 & FAIL:Squares \\
\hline
\end{tabular}

Model failures - Legend

:Delta ... Differential AIC/BIC criterion greater than 5

:Weight ... Akaike / Bayesian weight lower than 0.01

:Squares ... Relative sum of squared deviations greater than 1.05 
Model Discrimination Analysis - Conclusions

2 Different candidate models pass all acceptance criteria:

\begin{tabular}{rrrrrr}
\hline task no. & model ID & $n_{P}$ & $\mathrm{SSQ}_{r}$ & $\Delta \mathrm{AIC}$ & $\Delta \mathrm{BIC}$ \\
\hline 1 & $\mathrm{R} 1 \mathrm{~S}$ & 11 & 1 & 0 & 1.37715 \\
2 & $\mathrm{R} 1 \mathrm{~F}$ & 10 & 1.03748 & 1.26724 & 0 \\
\hline
\end{tabular}

Acceptable parameters

\begin{tabular}{llrrr}
\hline Model & Parameter & Value & Low & High \\
\hline R1S & ka.I & 3.41 & 2.5 & 5.24 \\
& kd.I & 0.00845 & 0.0057 & 0.0137 \\
R1F & kd.I & 0.0268 & 0.0247 & 0.0291 \\
\hline
\end{tabular}

